\title{
Dynamic Analysis and Circuit Realization of a Novel No-Equilibrium 5D Memristive Hyperchaotic System with Hidden Extreme Multistability
}

\author{
Qiuzhen Wan $\mathbb{D}^{1},{ }^{1}$ Zhaoteng Zhou $\mathbb{D}^{1},{ }^{1}$ Wenkui Ji $\mathbb{D},{ }^{1}$ Chunhua Wang, ${ }^{2}$ and Fei Yu $\mathbb{D}^{3}$ \\ ${ }^{1}$ Hunan Provincial Key Laboratory of Intelligent Computing and Language Information Processing, Hunan Normal University, \\ Changsha 410081, China \\ ${ }^{2}$ College of Computer Science and Electronic Engineering, Hunan University, Changsha 410082, China \\ ${ }^{3}$ School of Computer and Communication Engineering, Changsha University of Science and Technology, Changsha 410114, China
}

Correspondence should be addressed to Qiuzhen Wan; wanqiuzhen@sina.com

Received 10 July 2020; Revised 9 September 2020; Accepted 13 October 2020; Published 9 November 2020

Academic Editor: Akif Akgul

Copyright (C) 2020 Qiuzhen Wan et al. This is an open access article distributed under the Creative Commons Attribution License, which permits unrestricted use, distribution, and reproduction in any medium, provided the original work is properly cited.

In this paper, a novel no-equilibrium 5D memristive hyperchaotic system is proposed, which is achieved by introducing an ideal flux-controlled memristor model and two constant terms into an improved $4 \mathrm{D}$ self-excited hyperchaotic system. The system parameters-dependent and memristor initial conditions-dependent dynamical characteristics of the proposed memristive hyperchaotic system are investigated in terms of phase portrait, Lyapunov exponent spectrum, bifurcation diagram, Poincaré map, and time series. Then, the hidden dynamic attractors such as periodic, quasiperiodic, chaotic, and hyperchaotic attractors are found under the variation of its system parameters. Meanwhile, the most striking phenomena of hidden extreme multistability, transient hyperchaotic behavior, and offset boosting control are revealed for appropriate sets of the memristor and other initial conditions. Finally, a hardware electronic circuit is designed, and the experimental results are well consistent with the numerical simulations, which demonstrate the feasibility of this novel 5D memristive hyperchaotic system.

\section{Introduction}

As an important branch of nonlinear science, chaos has undergone great evolution and development since the first three dimensional (3D) system showing butterfly-shaped chaotic attractor was reported by Lorenz in 1963 [1-3]. Compared with chaos, hyperchaos possesses more randomness, complex dynamical behavior, and unpredictability with at least two positive Lyapunov exponents (LEs). Numerous examples of novel hyperchaotic systems [4-9] can be intensively discovered, and they have been widely applied in many fields, such as cryptography [10], neural network [11], synchronization $[12,13]$, and secure communications $[14,15]$.

The memristor that had been postulated as the fourth basic circuit element in 1971 by Chua [16] is a two-terminal passive electronic element described by nonlinear constitutive relation of charge $q$ and flux $\varphi$ and was for the first time fabricated in 2008 by Williams's group of HP Labs [17]. Currently, the modeling of basic memristor circuits $[18,19]$, the physical realization of all kinds of memristors and memristive systems $[20,21]$, the design and analysis of memristor-based application circuits [22, 23], etc. are attracting much attention in engineering communities. In particular, by introducing memristors with the intrinsic nonlinear characteristics into some existing dynamical systems, several memristor-based chaotic/hyperchaotic systems are proposed and applied [24-27]. These memristor-based chaotic/hyperchaotic systems could exhibit numerous nonlinear dynamical behaviors, such as hidden or self-excited attractors, and coexisting multiple [28] or coexisting infinitely many attractors $[29,30]$.

Hidden attractor is a new type of attractors and has received considerable interest recently $[31-35]$. The 
significant difference between self-excited attractor and hidden attractor is that the basin of attraction with selfexcited attractor is associated with one or more unstable equilibrium points, while that of hidden attractor does not intersect with any small neighborhoods of unstable equilibrium points. The hidden attractor exists in some special chaotic systems, including ones with only stable equilibrium points $[36,37]$, with an infinite number of equilibrium points [38, 39], or without equilibrium points [40, 41]. Since hidden attractor systems have neither heteroclinic nor homoclinic orbits, there is no way to verify the chaos state by conventional Shilnikov criteria [42]. However, there has been significant interest in studying hidden attractors because they play an important role in both academic significance and practical engineering applications [43]. For example, they allow unexpected and potentially disastrous responses to perturbations in a structure like an airplane wing or a bridge [44].

Multistability, meaning the coexistence of multiple attractors that rely on the different initial conditions and the same set of system parameters, is a fantastic physical phenomenon [45-47]. Multistability makes a nonlinear dynamical system offer great flexibility and exhibits a plentiful diversity of stable states of the system. In particular, when the number of coexisting attractors tends to infinity, the coexistence of infinitely many attractors is called extreme multistability. The emergence of extreme multistability is usually accompanied by other interesting dynamical behaviors just like transient behavior [48-50]. Since multistability can be taken as an additional source of randomness used for many information engineering applications [51] or used for image processing [52], it is attractive to seek a memristor-based hyperchaotic system that has the striking dynamical behavior of extreme multistability.

Motivated by the abovementioned considerations, a novel no-equilibrium $5 \mathrm{D}$ memristive hyperchaotic system is proposed in this paper, which is achieved through introducing an ideal flux-controlled memristor model and two constant terms into an improved 4D self-excited hyperchaotic system by [53]. From the physical circuit realization point of view, the corresponding 5D memristive hyperchaotic circuit is achieved by utilizing a memristor to substitute a linear coupling resistor and adding DC sources to realize two constant terms. The dynamical properties of the proposed system are investigated in terms of phase portrait, Lyapunov exponent (LE) spectrum, bifurcation diagram, Poincaré map, time series, and so on. To the best of the authors' knowledge, such a 5D memristive hyperchaotic system without equilibrium points exhibiting the striking phenomena of hidden extreme multistability, transient hyperchaotic behavior, and offset boosting control that has hardly been reported in the published literature until now, to a certain extent, is worthy of further detailed discussion.

The remaining sections of this paper are organized as follows. Section 2 briefly introduces how to obtain the mathematical model of the novel 5D memristive hyperchaotic system. The complex and abundant dynamical properties of this novel 5D memristive hyperchaotic system including hidden hyperchaos, extreme multistability, transient hyperchaotic behavior, and offset boosting control are analyzed numerically and theoretically in Section 3. In Section 4, the hardware electronic circuit is designed and the experimental results are presented to show its feasibility. Finally, the conclusions are summarized in Section 5.

\section{The Memristor and Novel $5 \mathrm{D}$ Memristive Hyperchaotic System}

The memristor is a two-terminal passive element with variable resistance called memductance $W(\varphi)$ or memristance $M(q)$. The memristance $M(q)$ described by nonlinear function $M(q)=\mathrm{d} \varphi(q) / \mathrm{d} q$ depends on the amount of charge $q$ that has passed through the memristor in a given direction. In other words, the memristor has the distinctive ability to memorize the past quantity of charge. The voltage-current $(v-i)$ characteristic curve of a memristor can exhibit a pinched hysteresis loop whose shape varies with the frequency. Let us consider an ideal flux-controlled memristor model $W(\varphi)$ in this paper, which can be described as

$$
\left\{\begin{array}{l}
\dot{i}=W(\varphi) v, \\
\dot{\varphi}=v, \\
W(\varphi)=\frac{\mathrm{d} q(\varphi)}{\mathrm{d} \varphi}=\alpha+\beta \varphi^{2},
\end{array}\right.
$$

where parameters $\alpha$ and $\beta$ are positive constants. Here, we just use the mathematical expression of the ideal fluxcontrolled memristor and neglect the physical significance of its variables.

Using simple feedback control techniques, by adding an additional state variable $w$ to the second equation of the wellknown Lorenz system, a 4D hyperchaotic system by [53] can be described as

$$
\left\{\begin{array}{l}
\dot{x}=a(y-x), \\
\dot{y}=b x-y-x z+w, \\
\dot{z}=-c z+x y, \\
\dot{w}=-d w-k x+e x y,
\end{array}\right.
$$

where $a, b, c, d, e$, and $k$ are all system parameters and $x, y, z$, $w$ are four state variables. When $a, b, c, d \in R+, e \in R-, k \in R$, these system parameters can determine the dynamic behaviors and bifurcations of system (2) with three equilibrium points or a unique zero equilibrium point.

Based on the 4D hyperchaotic system (2), the aforementioned ideal flux-controlled memristor model is introduced to the first equation, and the flux $\varphi$ that passes through the memristor became a new state variable denoted as $U$. We consider $Y$ as the state voltage $v$ inputting to the memristor, and let $\rho$ be a positive parameter indicating the strength of the memristor. From the physical circuit realization point of view, this approach is equivalent to replacing the linear coupling resistor which emulates the constant coefficient of the variable $y$ in the first equation with the memristor. At the same time, the two constant terms $g$ and $h$ 
are added to the second and third equation, respectively. Meanwhile, a linear state variable term $y$ of system (2) is reduced in the second equation. As a result, a dimensionless state equation set of a novel 5D memristive hyperchaotic system is constructed and described as

$$
\left\{\begin{array}{l}
\dot{X}=\rho W(U) Y-a X, \\
\dot{Y}=b X-X Z+W+g, \\
\dot{Z}=-c Z+X Y-h, \\
\dot{W}=-d W-e X-f X Z \\
\dot{U}=Y,
\end{array}\right.
$$

where $X, Y, Z, W, U$ and $\rho, a, b, c, d, e, f, g, h$ are the new five state variables and the system parameters. Here, it can be seen that system (3) has fourteen terms, including four (three quadratics and one cubic) nonlinear terms. It is worth noting that the nonzero constant terms $g$ and $h$ are newly introduced, which can make the proposed 5D memristive hyperchaotic system have no equilibrium points. Therefore, the nonzero constant terms $g$ and $h$ are especially valuable for the emergence of hidden extreme multistability in the presented system.

\section{Dynamic Analysis and Numerical Simulation}

In this section, with the help of equilibrium points, phase portrait, LE spectrum, bifurcation diagram, Poincaré map, and time series, the striking and complex nonlinear dynamical behaviors of the novel 5D memristive hyperchaotic system are broadly investigated by numerical simulations, including hidden hyperchaotic attractor, hidden extreme multistability, transient hyperchaotic behavior, and offset boosting control.

\subsection{Equilibrium Points and Typical Hidden Hyperchaotic} Attractor. The proposed 5D memristive hyperchaotic system (3) has the same dissipativity as the original 4D hyperchaotic system (2). Obviously, due to the introduction of the constant terms $g$ and $h$, the novel 5D memristive hyperchaotic system (3) is asymmetrical. For calculating the equilibrium points of system (3), let $\dot{X}=\dot{Y}=\dot{Z}=\dot{W}=\dot{U}=0$; the equilibrium equations can be obtained:

$$
\left\{\begin{array}{l}
0=\rho W(U) Y-a X \\
0=b X-X Z+W+g, \\
0=-c Z+X Y-h, \\
0=-d W-e X-f X Z, \\
0=Y .
\end{array}\right.
$$

Since $g$ and $h$ are all nonzero parameters, obviously, from the first, fourth, and fifth equations of system (3), $X=Y=W=0$, which is inconsistent with the second equation, provided that $g \neq 0$. Therefore, it can be shown that system (3) has no real solutions and thus system (3) has no equilibrium points, which is completely different from system (2) with three equilibrium points. As a result, the generating attractors of system (3) are all hidden, and the Shilnikov criteria cannot be adopted to explain its chaotic behavior because it has neither heteroclinic or homoclinic orbits.

Consider that the typical system parameters given in system (3) are chosen as $\rho=25, a=20, b=30, c=4, d=0.2$, $e=4, f=0.1, g=1, h=1, \alpha=1, \beta=0.02$, and the initial conditions $(X(0), Y(0), Z(0), W(0), U(0))$ are assigned as $(1$, $0,1,0,1)$; the proposed $5 \mathrm{D}$ memristive system exhibits a double-wing hyperchaotic hidden attractor, in which the projections of phase portraits on $X-Z, Y-Z, X-Y, X-W, Y-W$ and $Y-U$ planes are shown in Figures $1(\mathrm{a})-1(\mathrm{f})$, respectively. As can be seen from Figure 1, the proposed system has more complex attractor structure than system (2) presented by [53]. Correspondingly, the five LEs are calculated by Wolf's method with a step of 0.5 as $\mathrm{LE}_{1}=0.90134, \mathrm{LE}_{2}=0.2807$, $\mathrm{LE}_{3}=0.015517, \mathrm{LE}_{4}=-0.12893$, and $\mathrm{LE}_{5}=-25.2686$, and the Lyapunov dimension is $\mathrm{dL}=4.0423$, which indicates that system (3) is really a hyperchaotic system with two positive LEs.

The Poincare maps of the proposed system (3) projected on $X-Y$ plane with the $Z=30$ section and $Y-Z$ plane with the $X=Y$ section are shown in Figures 2(a)-2(b), respectively. Obviously, it can be seen that the Poincaré maps here contain an infinite number of dense points with fractal structures, which implies that the orbits of the attractors are continuously bifurcated and folded in different directions. It also illustrates that the system has hyperchaotic and extremely rich dynamical behavior.

3.2. Hidden Hyperchaotic Behavior Depending on System Parameters. The proposed memristive hyperchaotic system (3) has eleven system parameters which can affect the dynamical characteristics of the system. To analyze the generation of hidden hyperchaotic attractors of system (3) with respect to some system parameters, the LE spectrum and the corresponding bifurcation diagram as two useful tools are adopted. In this subsection, the system parameters $a$ and $b$ are adjusted, respectively, and the system parameters $\rho=25$, $c=4, d=0.2, e=4, f=0.1, g=1, h=1, \alpha=1, \beta=0.02$ as well as the initial conditions $(1,0,1,0,1)$ are fixed.

When system parameter $b=30$ and $a$ increases from 2 to 38 with a step size of 0.2 , the LEs of system (3) can be calculated as shown in Figure 3(a). It is worth mentioning that we have only sketched the first three LEs which are sufficient to qualify the behavior of the system. The last two LEs are not plotted because they are always large negative number. The corresponding bifurcation diagram of state variable $Y$ is given in Figure 3(b). With the change of system parameter $a$, from Figures 3(a) and 3(b), the system can be tracked in different states. The state of the system is related to the values of LEs. One zero and four negative LEs indicate the system is in periodic state. Two zero and three negative LEs indicate the system is in quasiperiodic state. One positive, one zero, three negative LEs and two positive, one zero, two negative LEs, respectively, indicate that the system is in chaotic and hyperchaotic states. Therefore, when system 


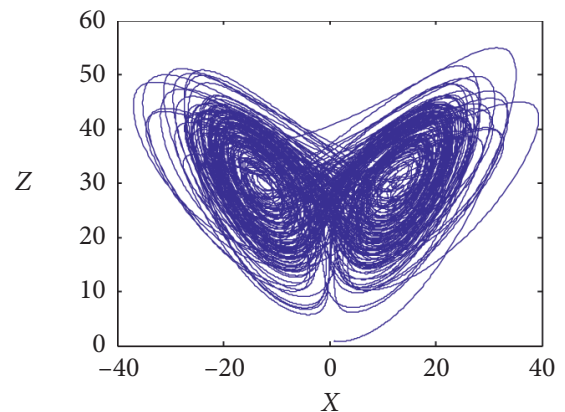

(a)

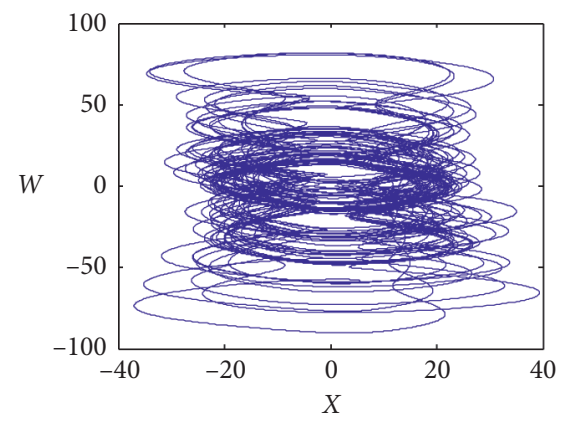

(d)

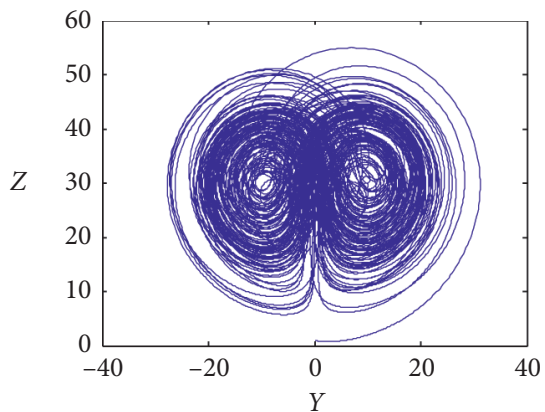

(b)

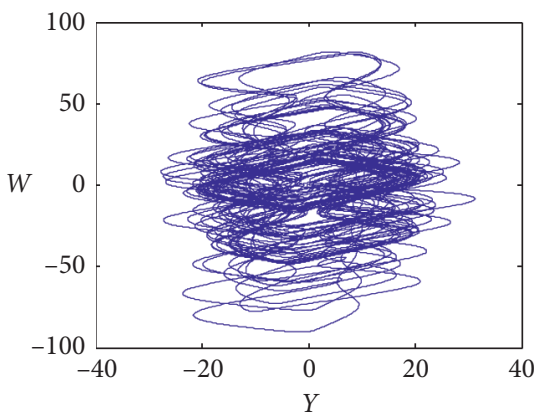

(e)

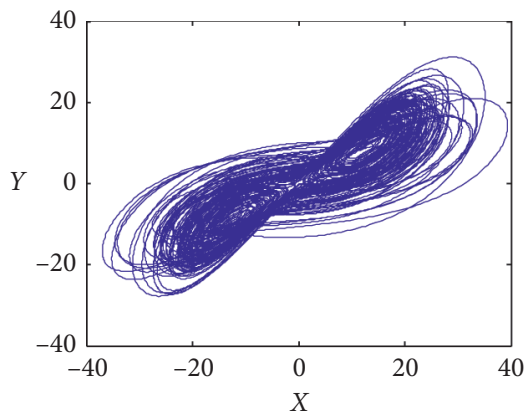

(c)

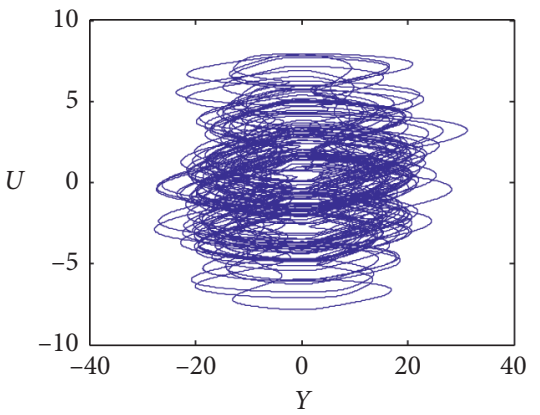

(f)

Figure 1: Different perspectives on the hyperchaotic attractor of system (3) without equilibrium.



(a)

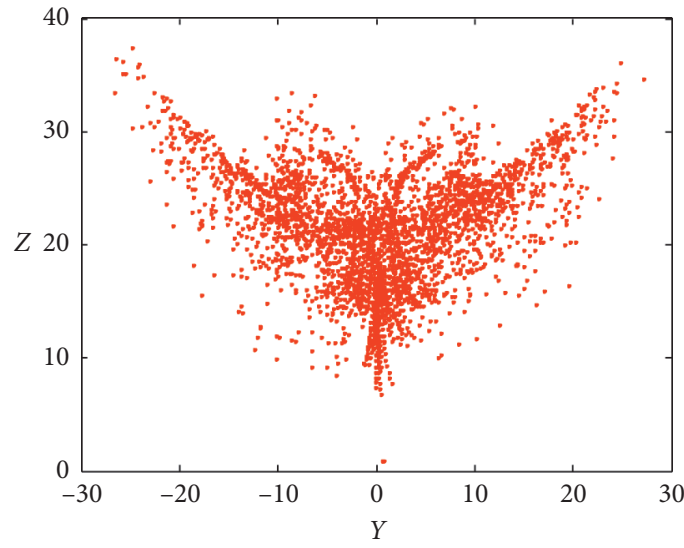

(b)

Figure 2: Poincaré maps of the hyperchaotic attractor of the system (a) on the $Z=30$ section and (b) on the $X=Y$ section.

parameter $a \in[2,3.5)$, the system is in weak chaotic state. When $a \in[3.5,12.1) \cup(33,38]$, the largest LE is a very small positive value or almost equals zero; then the system is in periodic or quasiperiodic limit cycle state. When $a \in[12.1$, $17.8]$, the system is in hyperchaotic state. When $a \in(17.8$, 33 ], the system is in chaotic state. There, periodic windows with zero largest LEs are also found in these chaotic regions. From the above numerical simulations, the proposed system (3) actually has rich hidden dynamics and can produce hyperchaos. It can be also shown that the bifurcation diagram matches well the LE spectrum.
Similarly, when $a=20$ and $b$ increases in the range of 0 to 60 , the first three LEs and the bifurcation diagram of state variable $Y$ are plotted in Figures 3(c) and 3(d). From Figures 3(c) and 3(d), the dynamical behaviors of system (3) can be clearly observed. With the change of system parameter $b$, the system can evolve into periodic state, hyperchaotic state, and chaotic state. Furthermore, for a wide range of system parameters $a$ and $b$, the results in Figure 3 have shown that the proposed $5 \mathrm{D}$ memristive system (3) indeed generates hidden hyperchaotic attractors. 


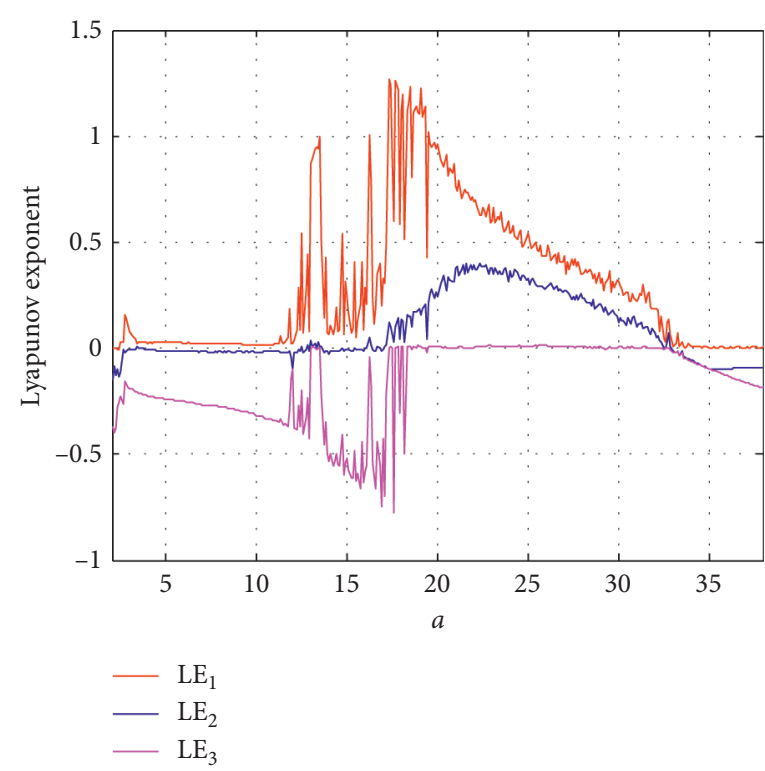

(a)

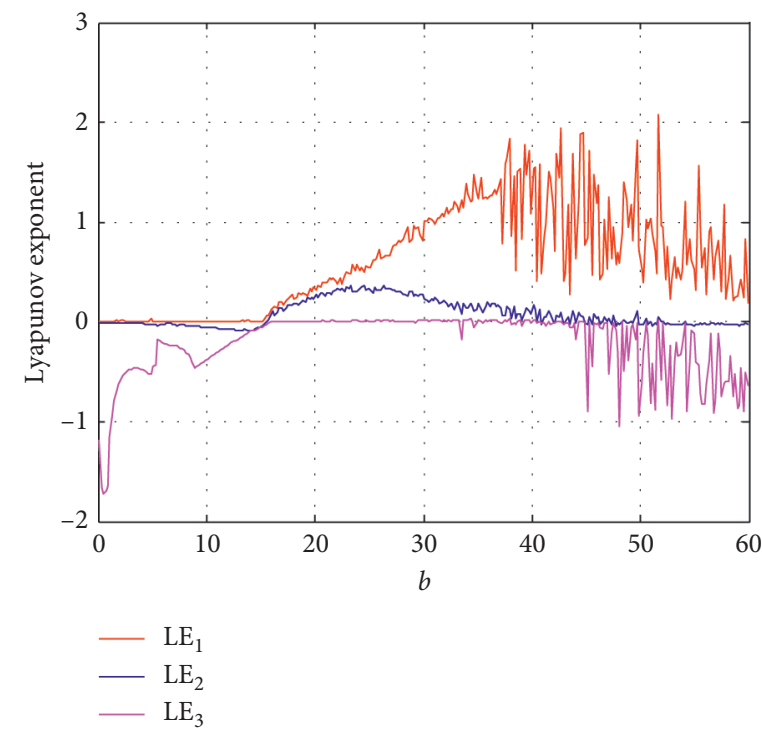

(c)

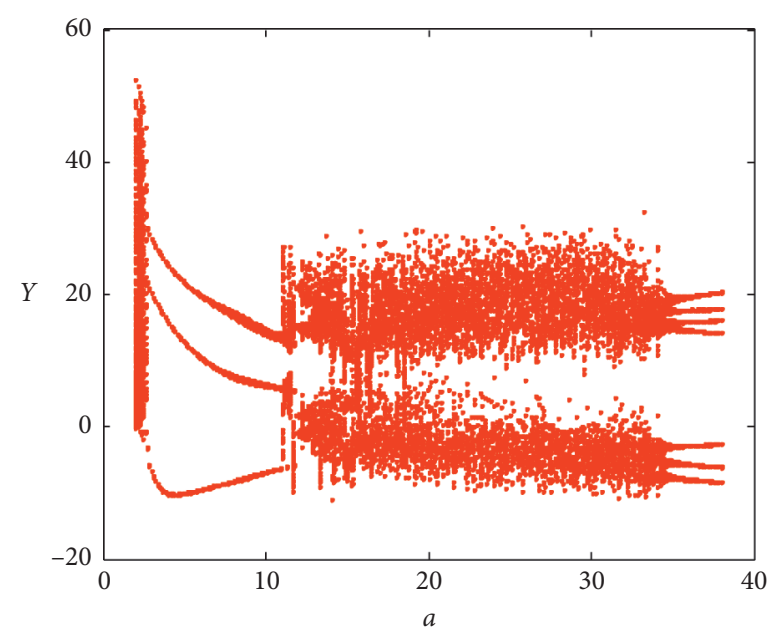

(b)

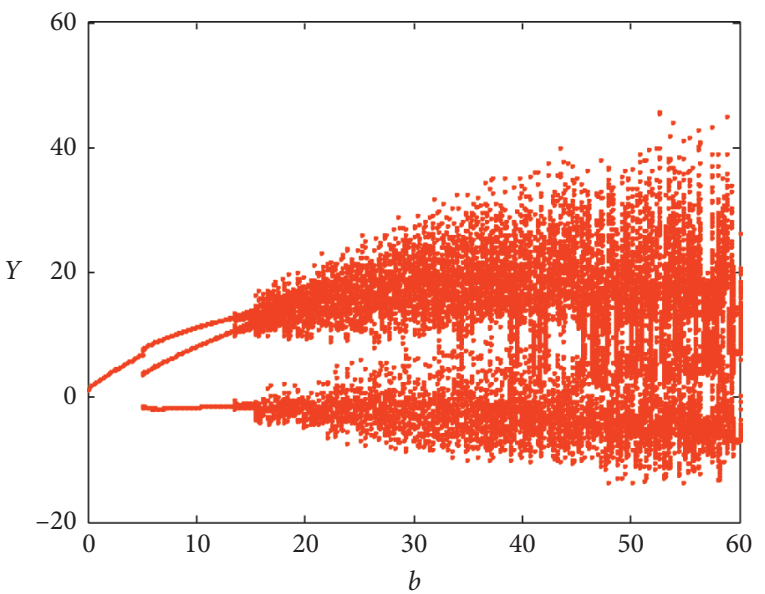

(d)

Figure 3: Hidden hyperchaotic dynamics: (a) and (c) are the first three LEs with the variation of parameters $a$ and $b$; (b) and (d) are the bifurcation diagrams of the state variable $Y$ with the variation of parameters $a$ and $b$.

More specifically, some evolutive orbits changing with system parameter $a$ in the $Y-Z$ plane are numerically displayed in Figures 4(a)-4(f). Figures 4(a) and 4(b) show the projections of some weak chaotic attractors, Figures 4(c) and $4(\mathrm{f})$ show the projections of periodic or quasiperiodic limit cycle, and Figures 4(d) and 4(e) show the projections of chaotic and hyperchaotic attractors.

\subsection{Hidden Extreme Multistability Relying on Memristor and} Other Initial Conditions. The dynamic characteristics of system (3) are affected not only by the system parameters, but also by the memristor and other initial conditions. In this subsection, we are going to explore hidden dynamical behaviors closely relying on the memristor and other initial conditions in the novel 5D memristive hyperchaotic system, in which the system parameters are kept constant. To a certain extent, initial conditions-dependent hidden dynamics just reveal the hidden extreme multistability phenomenon in system (3).

The typical system parameters are set as $\rho=25, a=20$, $b=30, c=4, d=0.2, e=4, f=0.1, g=1, h=1, \alpha=1, \beta=0.02$, and the initial conditions are fixed as $(1,0,1,0, U(0))$; here, the memristor initial condition $U(0)$ is taken as the bifurcation parameter. When $U(0)$ is gradually varying from -40 up to 40 , the first three LEs and the corresponding bifurcation diagram of the state variable $Y$ are plotted in Figures 5(a) and 5(b), respectively. It is seen that the 


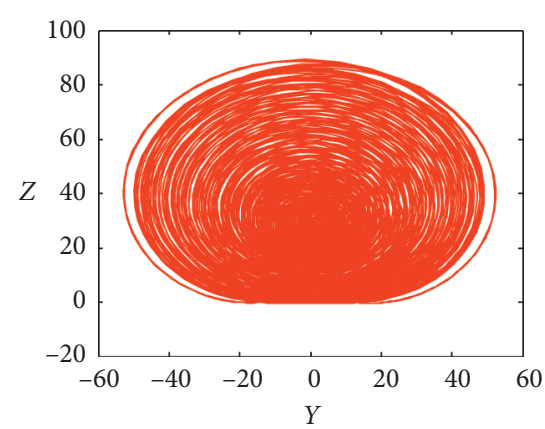

(a)

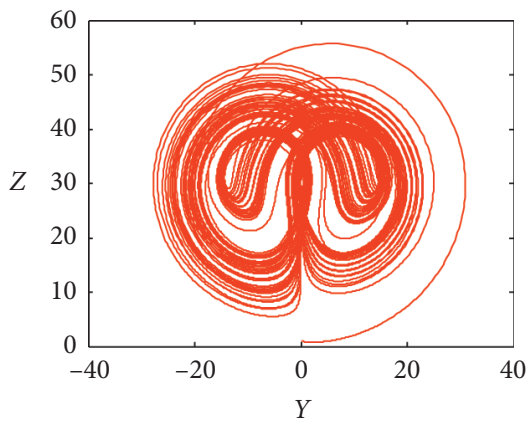

(d)

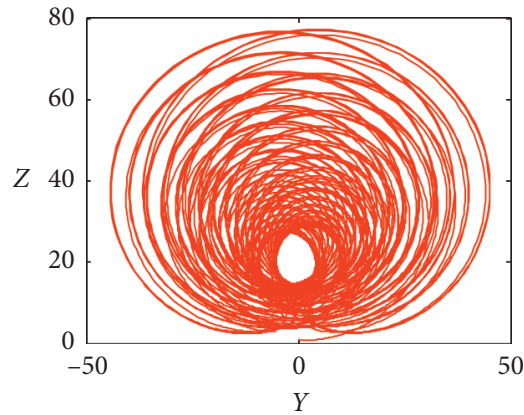

(b)

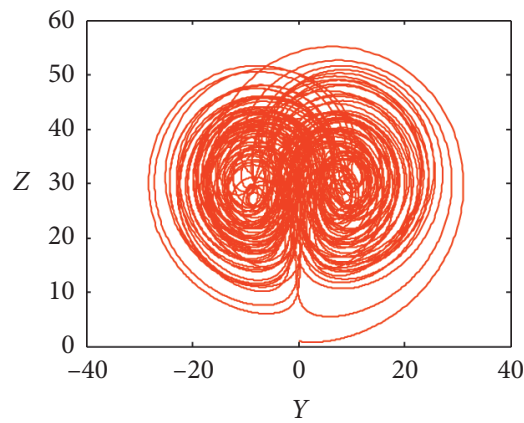

(e)

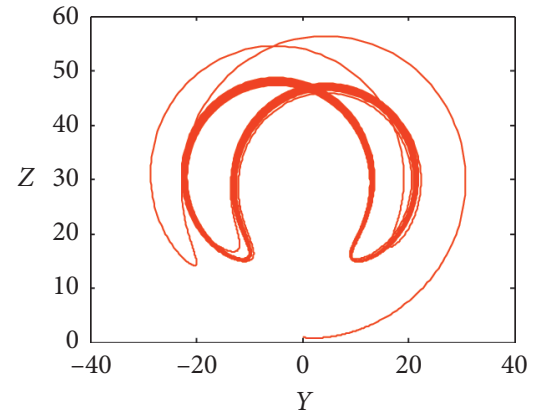

(c)

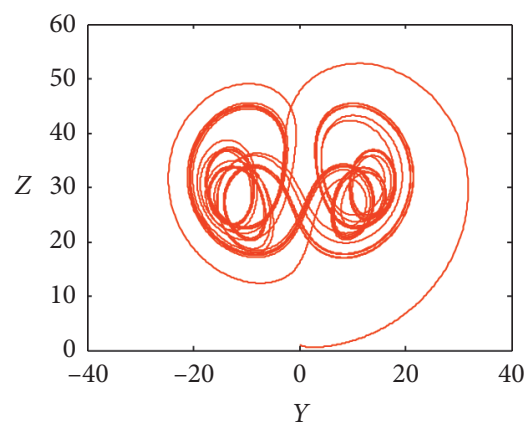

(f)

FIgURE 4: Typical phase portraits in the Y-Z plane with different $a$ values: (a) weak chaotic attractor $(a=2)$, (b) weak chaotic attractor $(a=2.6)$, (c) quasiperiodic limit cycle $(a=5)$, (d) chaotic attractor $(a=12.3)$, (e) hyperchaotic attractors $(a=18)$, (f) quasiperiodic limit cycle $(a=40)$.

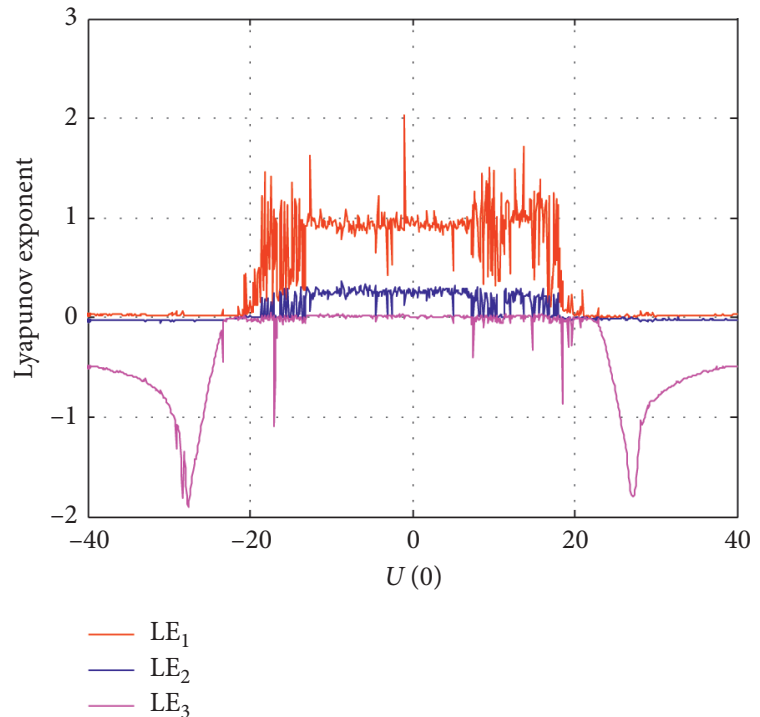

(a)

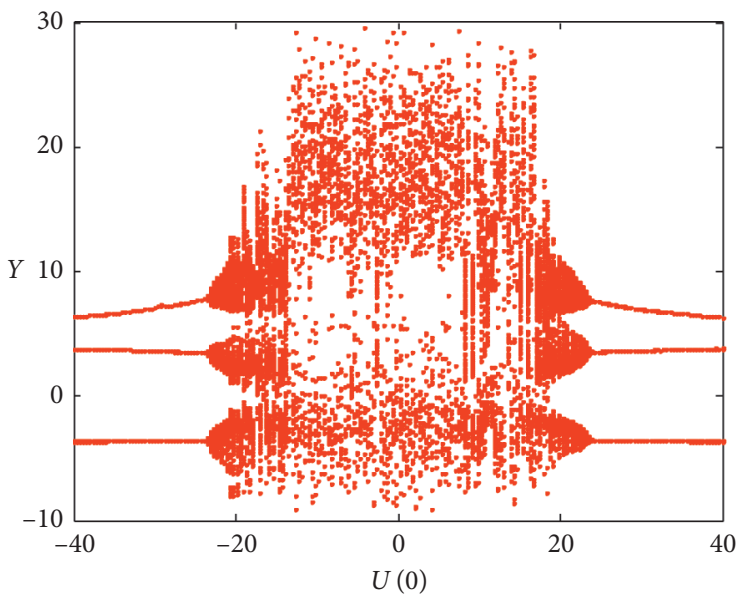

(b)

FIGURE 5: The occurrence of infinitely many hidden attractors of system (3) with respect to $U(0)$ : (a) the first three LEs, (b) the bifurcation diagram of the state variable $Y$.

bifurcation diagram matches well the LE spectrum. From Figure 5, it is easily found that the memristive system (3) can exhibit periodic, chaotic, hyperchaotic, and intermittent chaotic dynamics with the variation of the memristor initial condition $U(0)$. In Figure 5(a), we can notice the nearly symmetric phenomenon around the point 0 ; that is, the figure displayed from 0 to 40 is almost identical to the other half observed from -40 to 0 . When $U(0)$ is gradually increased from 0 , the bifurcation diagram shows that system (3) starts from hyperchaotic state to intermittent chaotic 
state, and then turns into periodic state through reversed period-doubling bifurcation route. The hyperchaotic behavior with two positive LEs is mainly located in the region of $[-18,18]$. At the same time, several narrow chaotic windows with one positive LE in the hyperchaotic region and some periodic windows with zero largest LEs in the chaotic region are also found.

In fact, the infinite number of coexisting hidden attractors is confirmed by Figure 5. As can be seen from Figure 6, several negative and positive memristor initial conditions are considered. When $U(0)$ is set to $-7,-20.5$, $-24.5,-30$, and -31.5 , respectively, the phase portraits of coexisting hidden multiple attractors in the $Y-Z$ plane and the $Y-Z-U$ space are displayed in Figures 6(a) and 6(b). Meanwhile, when $U(0)$ is set to $5,18.5,22.5,27.5$, and 32 , respectively, the phase portraits of coexisting hidden multiple attractors in the $Y-Z$ plane and the $Y-Z-U$ space are displayed in Figures 6(c) and 6(d). From Figure 6, it can be seen that the types of these hidden attractors are different from each other. Generally, Figure 6 clearly reveals the coexistence of an infinite number of hidden attractors with different dynamical behaviors, such as hyperchaos, chaos, and asymmetrical or symmetrical limit cycle, implying the emergence of extreme multistability in system (3).

On the other hand, when the system parameters of system (3) are kept unchanged, the initial conditions $(X(0)$, $Y(0), Z(0), W(0), U(0))$ are assigned as $(X(0), 0,1,0,1)$, and the other initial condition $X(0)$ is taken as the bifurcation parameter. When $X(0)$ is varied in the region of $[-100,100]$, the first three LEs and the corresponding bifurcation diagram of the state variable $Y$ are plotted in Figures 7(a) and $7(\mathrm{~b})$, respectively. With the variations of the other initial condition $X(0)$, it can be seen that system (3) has two states of chaotic attractor and periodic limit cycle. In the same way, several negative and positive initial conditions are considered according to Figure 7 . When $X(0)$ is set to $-40,-15,19$, 52 , respectively, the phase portraits of coexisting hidden multiple attractors in the $Y-Z$ plane are displayed in Figures 8(a) and 8(b). Similarly, we can observe an infinite number of coexisting attractors with different topological structures including both chaotic and periodic motions. As a result, it is remarked that when the memristor initial condition $U(0)$ and the other initial conditions of $X(0), Y(0)$, $Z(0)$, and $W(0)$ are assigned to different values, system (3) has completely different bifurcation behaviors, which further indicates that there is coexisting infinitely many attractors' behavior in system (3).

3.4. Transient Hyperchaotic Behavior. The interesting phenomenon of transient hyperchaotic behavior means that a nonlinear system behaves as hyperchaotic attractor in a finite time interval and then turns into a periodic or another hyperchaotic/chaotic attractor with the time evolution, which has been observed in many nonlinear dynamical systems. Interestingly, system (3) exhibits complex transient hyperchaotic behavior when choosing appropriate system parameters and initial conditions.
Firstly, we just fix the system parameter $\rho=27$, while the other system parameters and initial conditions of system (3) are kept unchanged and selected as the typical values. The phase portraits and the corresponding time-domain waveform are depicted in Figures 9(a) and 9(b), where the trajectories of system (3) have a transition from a transient hyperchaos to another steady hyperchaos with two totally different amplitudes of the state variable $X$, and the LEs are calculated as $\mathrm{LE}_{1}=1.073, \mathrm{LE}_{2}=0.1981, \mathrm{LE}_{3}=0.0177$, $\mathrm{LE}_{4}=-0.0973$, and $\mathrm{LE}_{5}=-25.3915$. Secondly, by selecting $U(0)=-14$ and keeping the other initial conditions and system parameters unchanged, the trajectories of system (3) change from a transient hyperchaotic attractor to another steady chaotic attractor with the time evolution. At the moment, the phase portraits and time-domain waveform are shown in Figures 9(c) and 9(d), in which we can expressly see that the two dynamic amplitudes of the state variable $X$ are quite different. The corresponding LEs are calculated as $\mathrm{LE}_{1}=0.5624, \quad \mathrm{LE}_{2}=0, \quad \mathrm{LE}_{3}=-0.0242, \quad \mathrm{LE}_{4}=-1.9205$, and $\mathrm{LE}_{5}=-22.8422$, implying that system (3) oscillates in chaotic mode. Therefore, the results shown in Figure 9 demonstrate that transient hyperchaotic behavior occurs in system (3), which leads to the generation of two attractors with different amplitudes and topological structures.

3.5. Offset Boosting Control. In this subsection, another distinctive trait of the novel no-equilibrium 5D memristive hyperchaotic system is the offset boosting control. When a state variable has a single occurrence in the right hand side of a nonlinear system, then adding a new constant to that state variable might produce an offset and thus give the freedom to transform the chaotic signal from unipolar to bipolar or vice versa. This feature has been reported in few chaotic systems previously $[54,55]$. From the perspective of engineering application, the offset boosting control is important since it represents a useful alternative to control the amplitude and voltage level of the state variable for signal transformation and transmission.

It is particularly exciting for us that the proposed $5 \mathrm{D}$ memristive hyperchaotic system (3) can be offset-boosted in at least two manners. Firstly, for $c=f=0$, the state variable $Z$ appears just once in the second equation of system (3). The state variable $Z$ is flexibly offset-boosted by replacing $Z$ with $Z+n$, where $n$ is a new constant named as a controller. Now the equations of system (3) can be rewritten as

$$
\left\{\begin{array}{l}
\dot{X}=\rho W(U) Y-a X, \\
\dot{Y}=b X-X(Z+n)+W+g, \\
\dot{Z}=X Y-h, \\
\dot{W}=-d W-e X, \\
\dot{U}=Y .
\end{array}\right.
$$

When the other system parameters are $\rho=25, a=20$, $b=5, d=0.2, e=4, g=1, h=80, \alpha=1, \beta=0.02$, and the initial conditions are selected as $(1,0,1,0,1)$, we can acquire a hyperchaotic hidden attractor. By selecting three different 


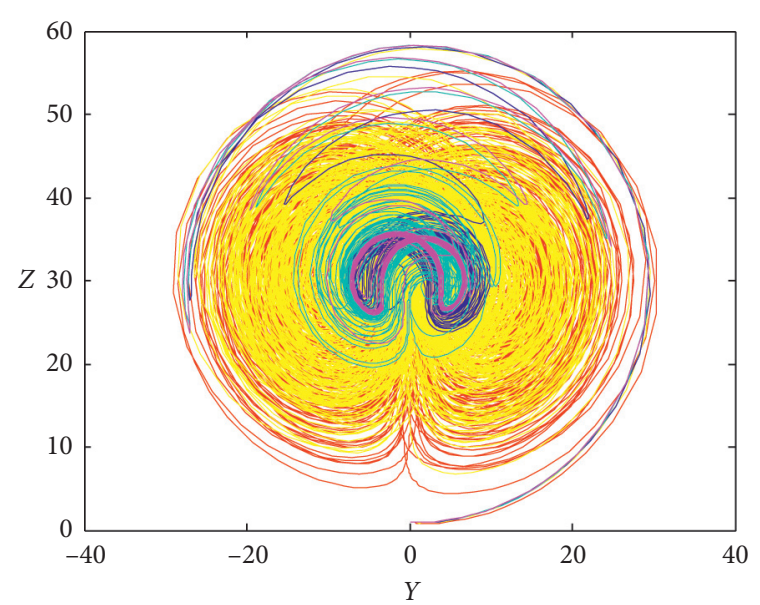

(a)

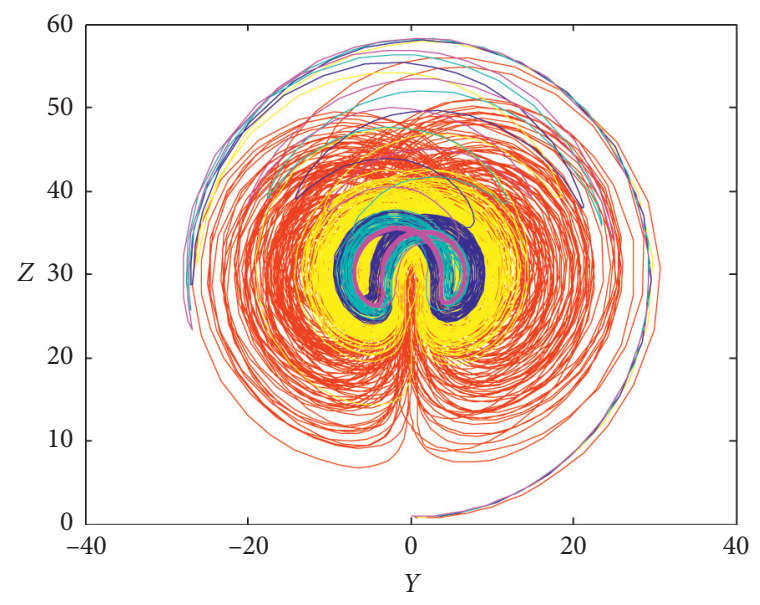

(c)

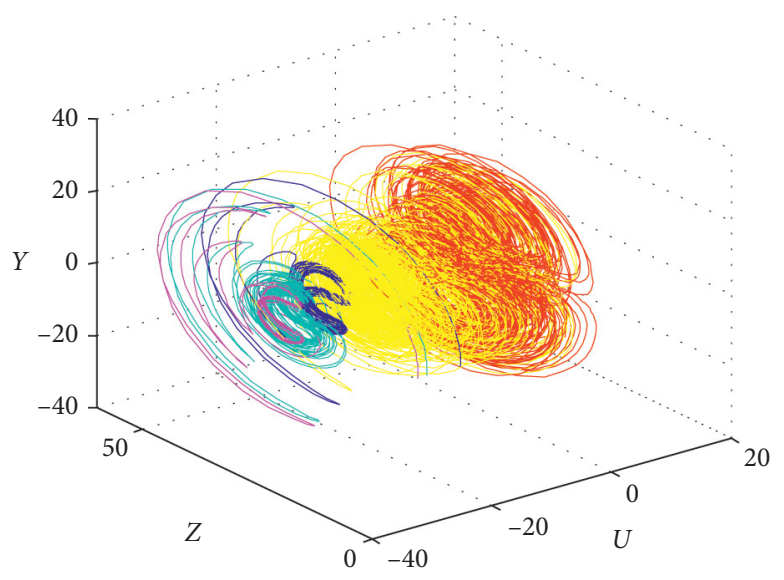

(b)

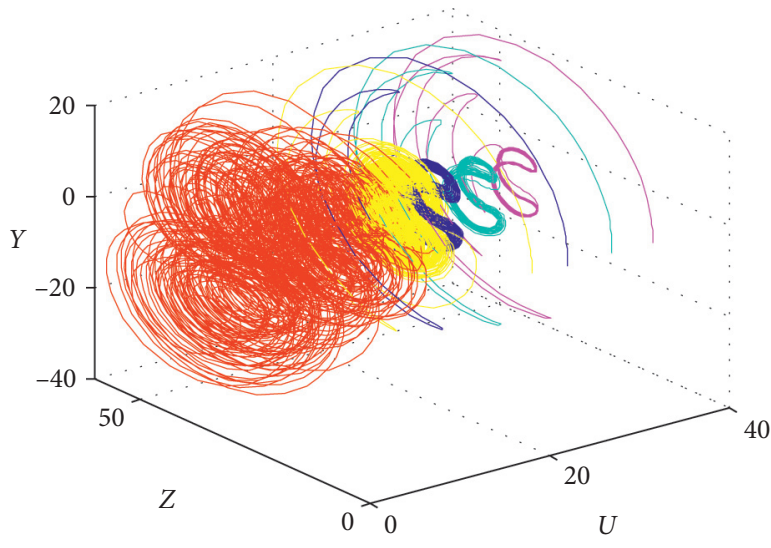

(d)

FiguRE 6: The phase portraits of the occurrence of infinitely many hidden attractors in the $Y$ - $Z$ plane and the $Y$ - $Z$ - $U$ space with different negative and positive $U(0)$. (a), (b) $U(0)=-7$ (red), hyperchaos; $U(0)=-20.5$ (yellow), chaos; $U(0)=-24.5$ (blue) or $U(0)=-30$ (cyan), asymmetrical limit cycle; $U(0)=-31.5$ (magenta), symmetrical limit cycle. (c), (d) $U(0)=5$ (red), hyperchaos; $U(0)=18.5$ (yellow), chaos; $U(0)=22.5$ (blue) or $U(0)=27.5$ (cyan), asymmetrical limit cycle; $U(0)=32$ (magenta), symmetrical limit cycle.

values of $n$, for $n=-30$ (green), $n=0$ (blue), and $n=30$ (magenta), the different positions with the same shape on the $X-Z$ and $Y-Z$ planes of the new hyperchaotic hidden attractors are exhibited in Figures 10(a) and 10(b). From Figure 10, we can find that the negative or positive value of the controller $n$ will boost the state variable $Z$ in the positive or negative direction, respectively. Figures $10(\mathrm{c})$ and $10(\mathrm{~d})$ also show that the state variable $Z$ is boosted from a hyperchaotic bipolar signal to a hyperchaotic unipolar signal by changing the value of the controller $n$, but the LEs remain constant, which indicated that the controller $n$ cannot influence the dynamics of system (5).

Secondly, when setting $d=0$ and the other parameters as $\rho=25, a=20, b=30, c=4, e=4, f=0.1, g=1, h=1, \alpha=1, \beta$ $=0.02$ with the initial conditions unchanged, system (3) is reduced to system (6) and the state variable $\mathrm{W}$ can be easily boosted since it also appears just once in the second equation of system (3). Now the equations of system (3) can be rewritten as

$$
\left\{\begin{array}{l}
\dot{X}=\rho W(U) Y-a X, \\
\dot{Y}=b X-X Z+(W+m)+g, \\
\dot{Z}=-c Z+X Y-h, \\
\dot{W}=-e X-f X Z, \\
\dot{U}=Y .
\end{array}\right.
$$

Thus, the state variable $W$ is offset-boosted by replacing $W$ with $W+m$, where $m$ is a new constant. As depicted in Figure 11, various positions of the phase portraits of system (6) with hyperchaotic hidden attractors have been adjusted depending on different values of $m$ in the $X-W$ and $Y$-W planes, where the positive value of $m$ boosts the state variable $W$ in the negative direction or vice versa. Besides, as shown in Figure 11, the controller $m$ in the region of $[-60,60]$ can merely change the average value of the state variable $W$ and keep the other four state variables $(X, Y, Z$ and $U)$ unchanged. Therefore, the controller $m$ cannot influence the 


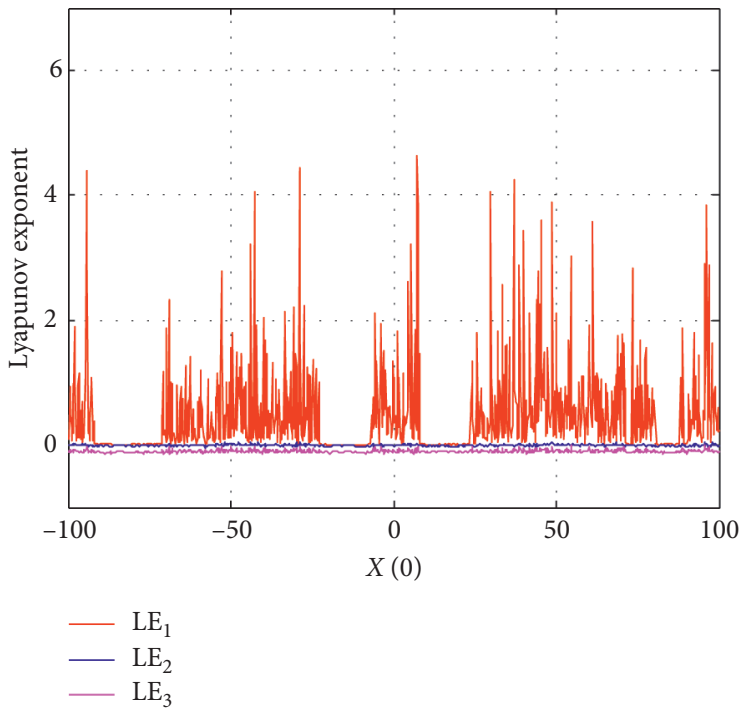

(a)

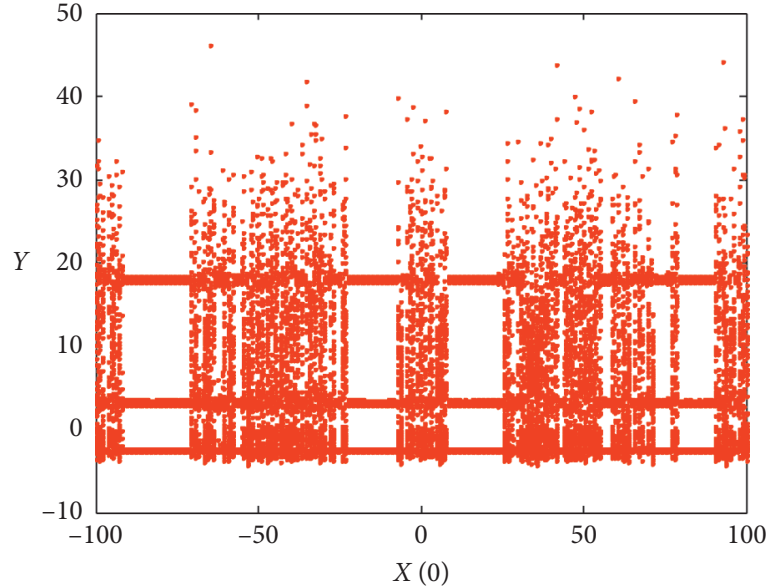

(b)

FIGURE 7: Dynamics with respect to the other initial condition $X(0)$ : (a) the first three LEs, (b) the bifurcation diagram of the state variable $Y$.

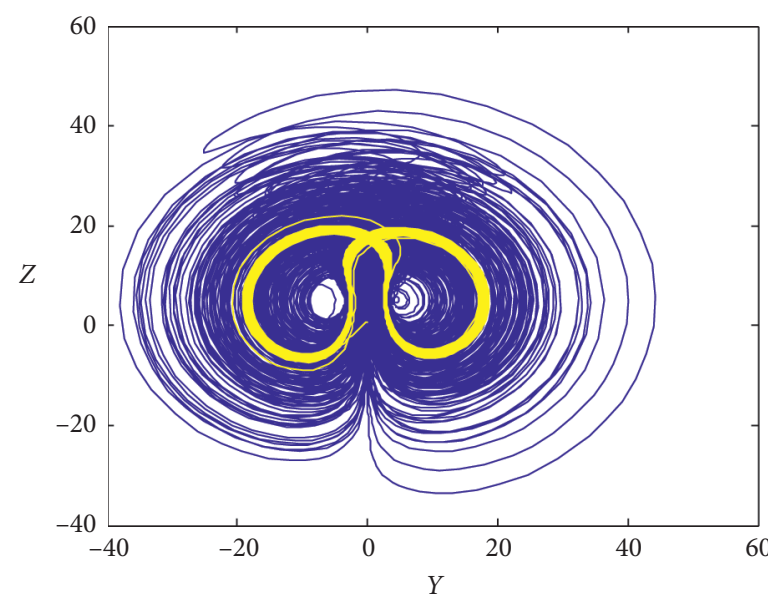

(a)

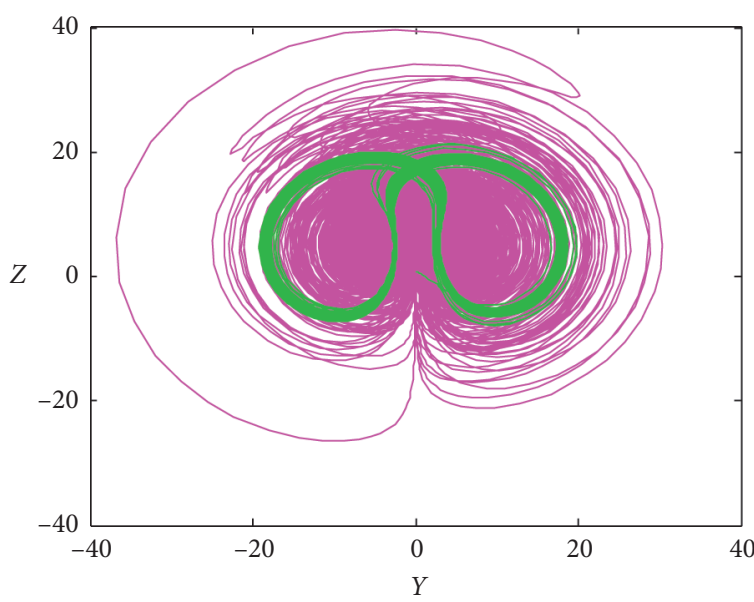

(b)

FIgURE 8: The phase portraits of coexisting hidden multiple attractors in the $Y$ - $Z$ plane with different negative and positive $X(0)$ values. (a) $X(0)=-40$ (blue), chaos; $X(0)=-15$ (yellow), limit cycle. (b) $X(0)=19$ (green), limit cycle; $X(0)=52$ (magenta), chaos.

dynamics of system (6) which results in the constant LEs as illustrated in Figure 11.

\section{Circuit Implementation and Experimental Results}

Generally, the hardware circuit implementation of chaotic system not only can validate the mathematical model but also has been used in engineering applications such as encryption, secure communication, and random signal generation. In this section, to obtain the hyperchaotic hidden attractor, Figure 12 shows a module-based analog circuit with off-the-shelf discrete components on a breadboard which is designed for implementation of system (3).
The operational amplifiers selected as LM741 and analog multipliers chosen as AD633JN versions of AD633 with $\pm 15 \mathrm{~V}$ power supplies are utilized. The basic operations of addition, subtraction, and integration are performed by the operational amplifiers with the associated resistors or capacitors. The multiplication operation is performed by the analog multipliers. Since AD633JN has an overall scale factor of $1 / 10 \mathrm{~V}$, a factor of $0.1 \mathrm{~V}$ needs to be put on each nonlinear term. As shown in Figure 12, by replacing the linear coupling resistor having $V_{A}$ and $V_{B}$ as terminals voltages with the memristor emulator, system (3) is easily implemented. The equivalent realization module of the fluxcontrolled memristor $W(U)$ is depicted in the dotted box of Figure 12, where $U$ is the inner state variable of capacitor voltage in the memristor. The DC sources of $V_{1}$ and $V_{2}$ are 


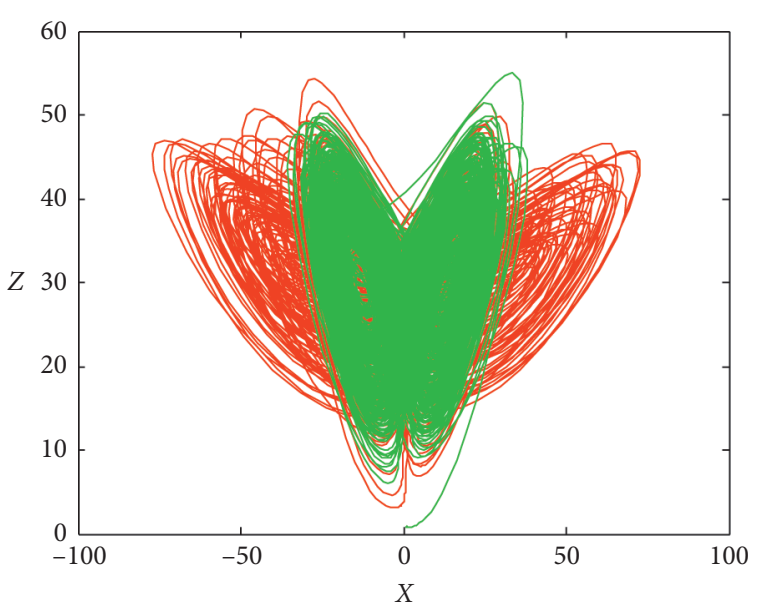

(a)

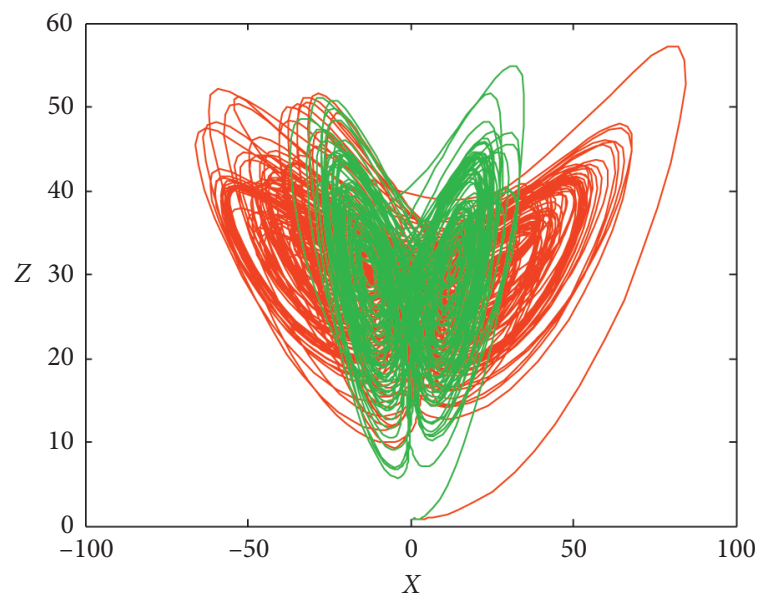

(c)

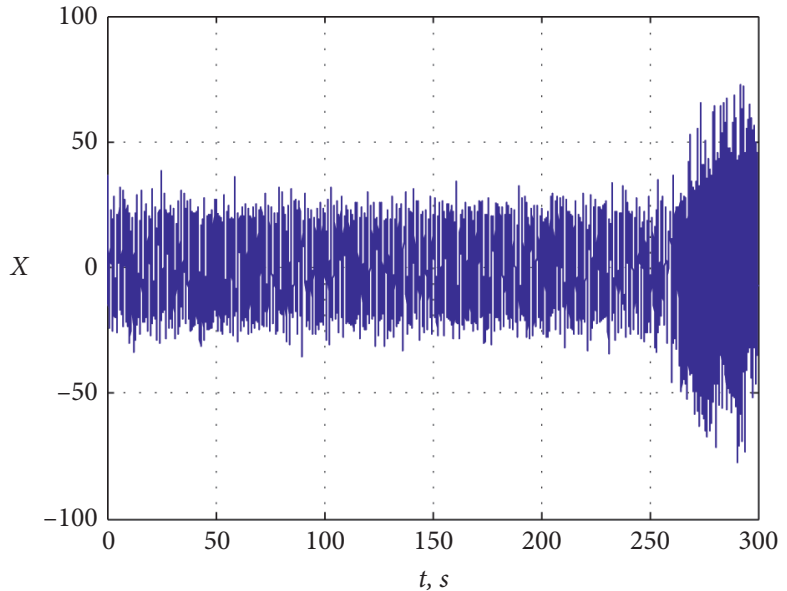

(b)

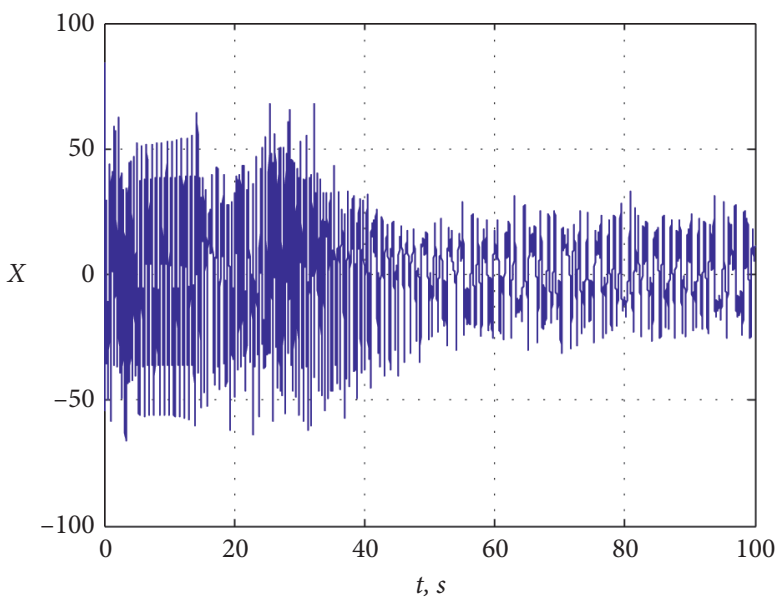

(d)

FIGURE 9: Transient hyperchaotic behavior: (a) and (c) are phase portraits; (b) and (d) are time-domain waveforms; the system parameter $\rho=27$ in (a) and (b), whereas the memristor initial condition $U(0)=-14$ in (c) and (d).

used to realize the constant terms $g$ and $h$ of system (3), respectively. As denoted in Figure 12, $X, Y, Z, W$, and $\mathrm{U}$ correspond to the voltages of $C_{1}, C_{2}, C_{3}, C_{4}$, and $C_{5}$, respectively. The initial conditions $(X(0), Y(0), Z(0), W(0)$, $U(0)$ ) of all the state variables of Figure 12 can be acquired by turning on the hardware circuit power supply again.

Note that all the state variables of system (3) exceed the saturation amplitude range of operational amplifiers LM741 and analog multipliers AD633JN; their values should be scaled down. According to system (3), by changing the state variables $(X, Y, Z, W, U)$ to $(10 X, 10 Y, 10 Z, 20 W, 2 U)$, system (3) can be expressed as

$$
\left\{\begin{array}{l}
\dot{X}=-a X+\rho \alpha Y+4 \rho \beta U^{2} Y, \\
\dot{Y}=b X-10 X Z+2 W+0.1 g, \\
\dot{Z}=-c Z+10 X Y-0.1 h, \\
\dot{W}=-d W-0.5 e X-5 f X Z, \\
\dot{U}=5 Y .
\end{array}\right.
$$

By applying the voltage-current relations of circuit elements, the Kirchhoff's circuit laws, and the circuit properties of operational amplifiers, the circuit state equations of Figure 12 can be described as

$$
\left\{\begin{array}{l}
\dot{X}=-\frac{1}{C_{1} R_{1}} X+\frac{1}{C_{1} R_{2}} Y+\frac{R_{22}}{100 C_{1} R_{3} R_{21}} U^{2} Y, \\
\dot{Y}=\frac{1}{C_{2} R_{4}} X-\frac{1}{10 C_{2} R_{5}} X Z+\frac{1}{C_{2} R_{6}} W+\frac{V_{1}}{C_{2} R_{7}}, \\
\dot{Z}=-\frac{1}{C_{3} R_{8}} Z+\frac{1}{10 C_{3} R_{9}} X Y-\frac{V_{2}}{C_{3} R_{10}}, \\
\dot{W}=-\frac{1}{C_{4} R_{11}} W-\frac{1}{C_{4} R_{13}} X-\frac{1}{10 C_{4} R_{12}} X Z \\
\dot{U}=\frac{1}{C_{5} R_{14}} Y
\end{array}\right.
$$




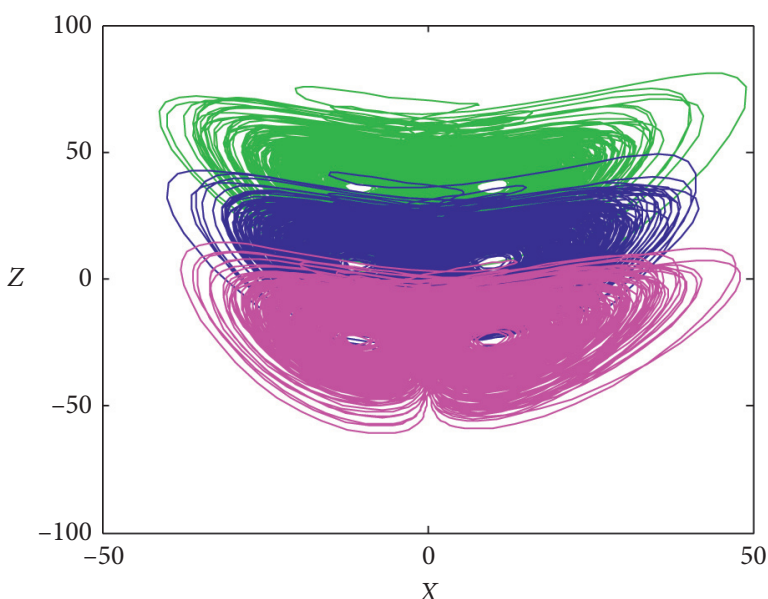

(a)

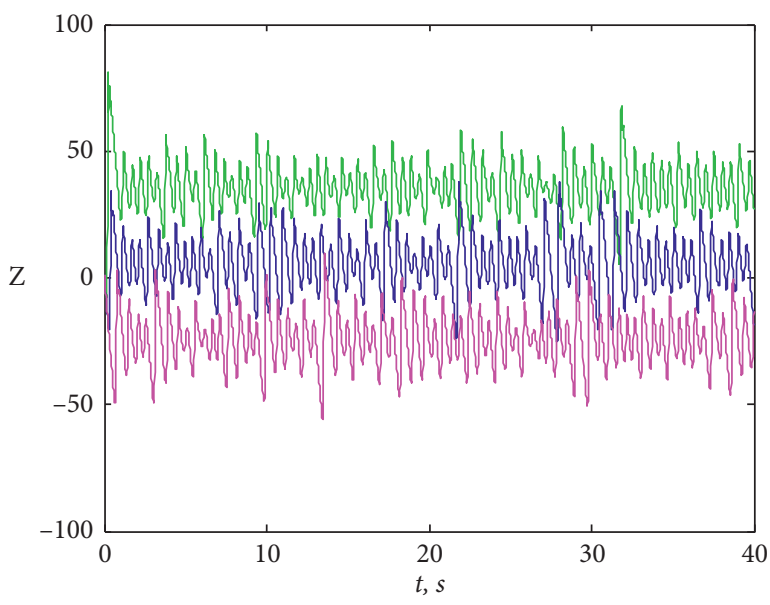

(c)

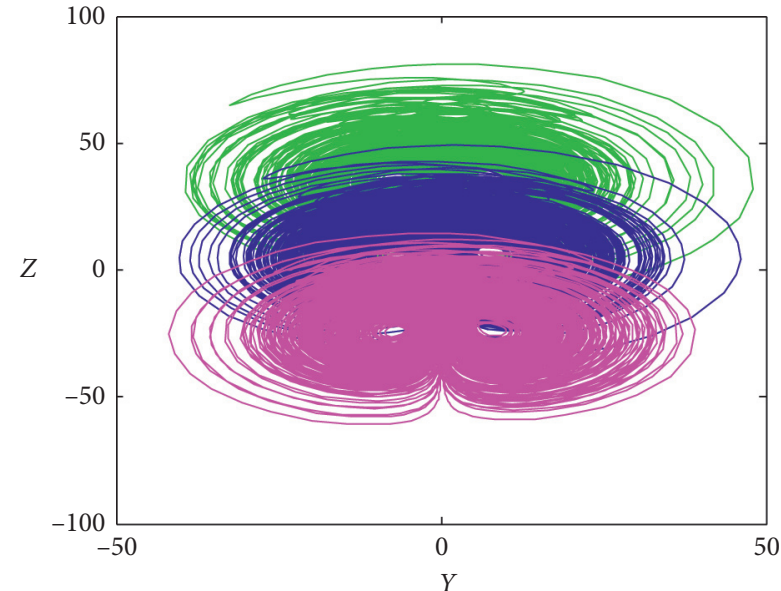

(b)

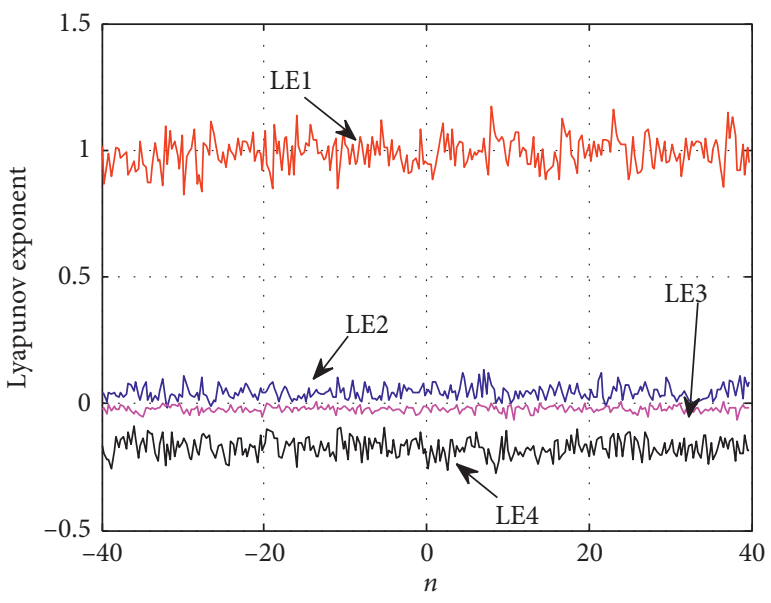

(d)

FIGURE 10: The offset boosting with $n=-30$ (green), $n=0$ (blue), and $n=30$ (magenta): (a) phase portrait in the $X$ - $Z$ plane, (b) phase portrait in the $Y$ - $Z$ plane, (c) time series of the variable $Z$, (d) LEs spectrum.

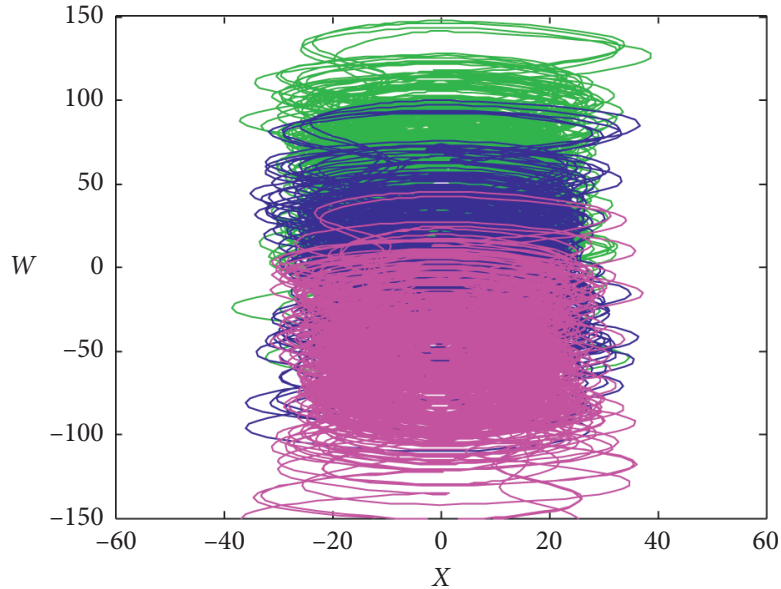

(a)

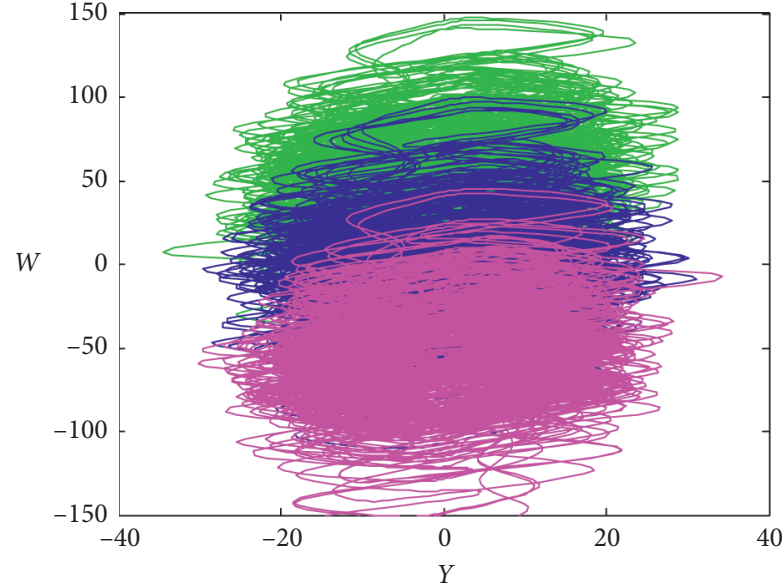

(b)

Figure 11: Continued. 


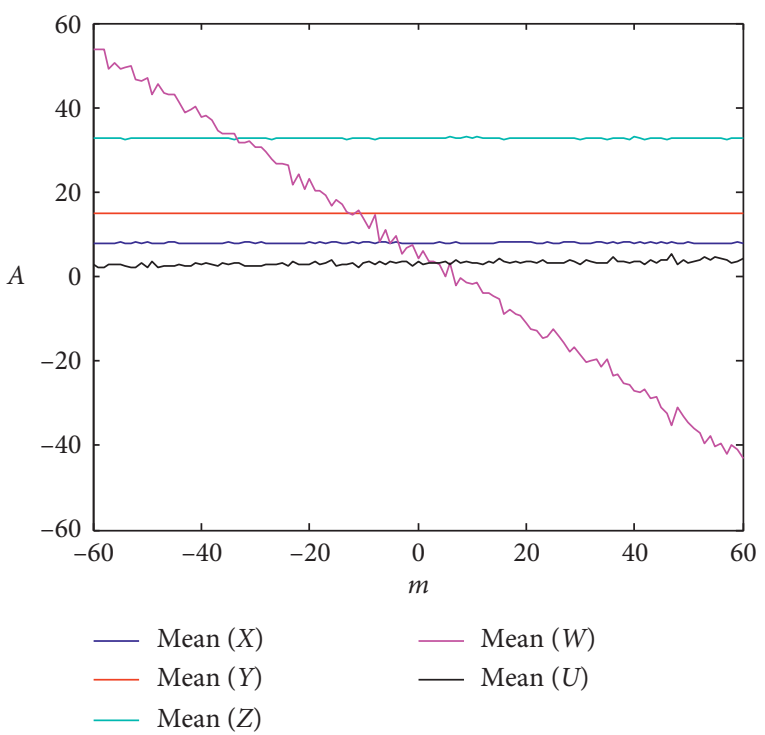

(c)

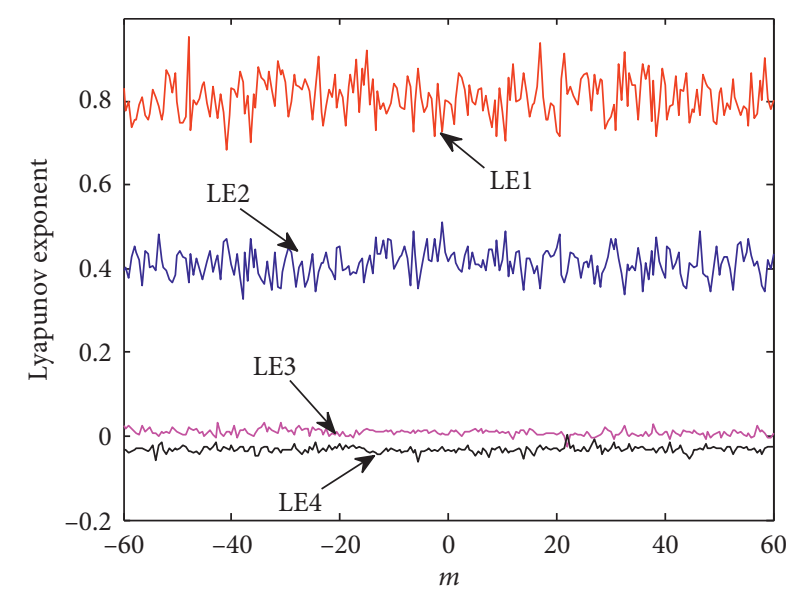

(d)

Figure 11: The offset boosting with $m=-55$ (green), $m=0$ (blue), and $m=55$ (magenta): (a) phase portrait in the $X$ - $W$ plane; (b) phase portrait in the $Y$-W plane; (c) the average values of the variables $X Y, Z, W$, and $U$; (d) LEs spectrum.

Comparing equation (7) with equation (8) and setting the corresponding coefficients to be equal, we can obtain

$$
\begin{aligned}
a & =\frac{1}{C_{1} R_{1}}, \\
\rho \alpha & =\frac{1}{C_{1} R_{2}}, \\
4 \rho \beta & =\frac{R_{22}}{100 C_{1} R_{3} R_{21}}, \\
b & =\frac{1}{C_{2} R_{4}}, \\
10 & =\frac{1}{10 C_{2} R_{5}}, \\
2 & =\frac{1}{C_{2} R_{6}}, \\
0.1 g & =\frac{V_{1}}{C_{2} R_{7}},
\end{aligned}
$$

$$
\begin{aligned}
c & =\frac{1}{C_{3} R_{8}}, \\
10 & =\frac{1}{10 C_{3} R_{9}}, \\
0.1 h & =\frac{V_{2}}{C_{3} R_{10}}, \\
d & =\frac{1}{C_{4} R_{11}}, \\
0.5 e & =\frac{1}{C_{4} R_{13}}, \\
5 f & =\frac{1}{10 C_{4} R_{12}}, \\
5 & =\frac{1}{C_{5} R_{14}} .
\end{aligned}
$$

Let us take $C_{1}=C_{2}=C_{3}=C_{4}=C_{5}=1 \mathrm{uF}$ and $V_{1}=V_{2}=1 \mathrm{~V}$; when $\rho=25, a=20, b=30, c=4, d=0.2, e=4, f=0.1, g=1$, $h=1, \alpha=1$, and $\beta=0.02$, the value of the integrating resistor can be obtained as $R_{1}=R_{3}=50 \mathrm{k} \Omega, \quad R_{2}=40 \mathrm{k} \Omega$, $R_{4}=33.333 \mathrm{k} \Omega, \quad R_{6}=R_{13}=500 \mathrm{k} \Omega, \quad R_{7}=R_{10}=10 \mathrm{M} \Omega$, 

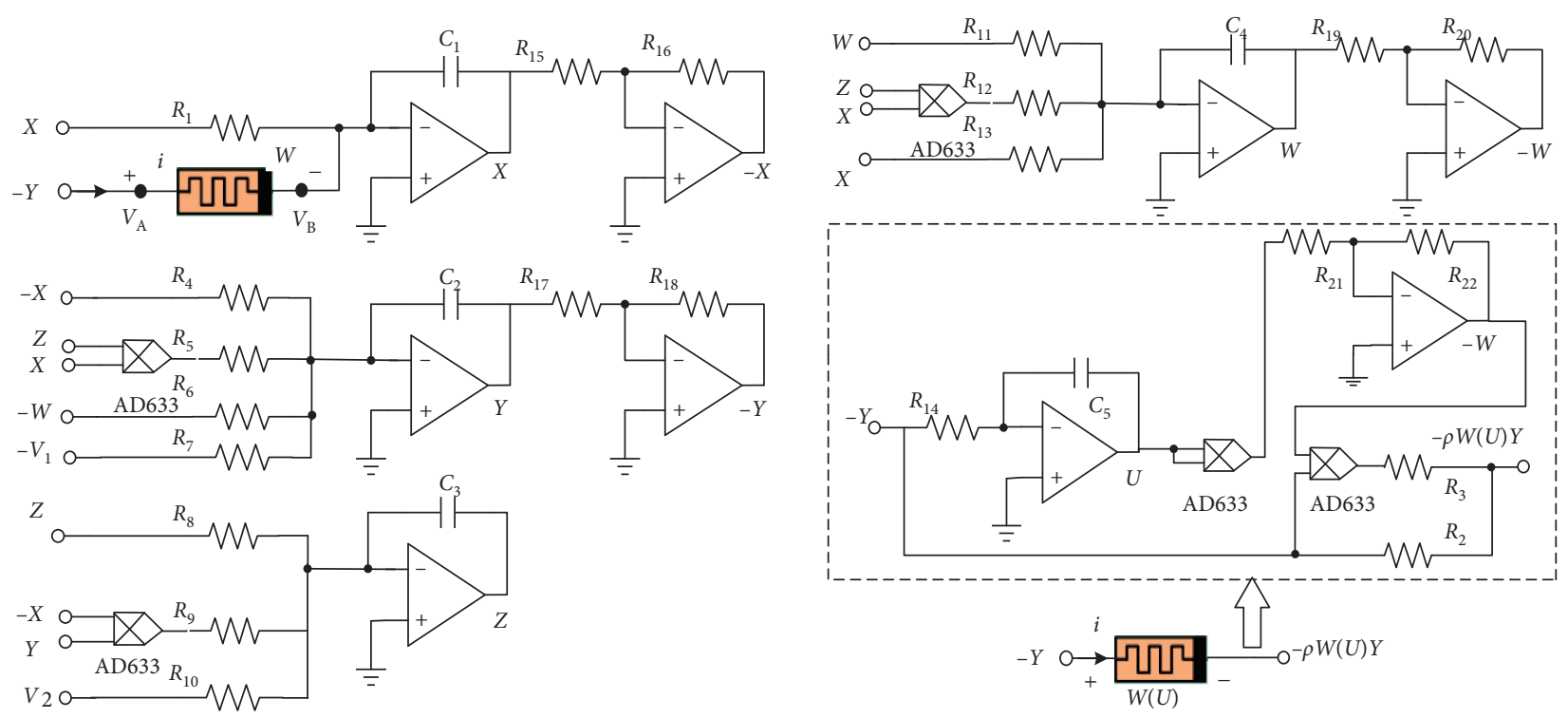

Figure 12: Circuit implementation of the proposed 5D memristive hyperchaotic system.

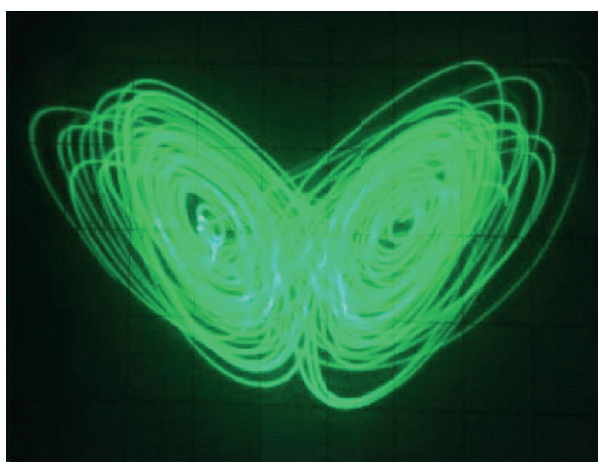

(a)

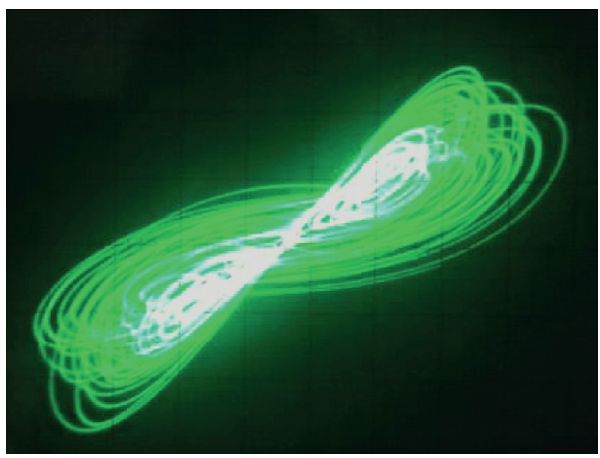

(c)

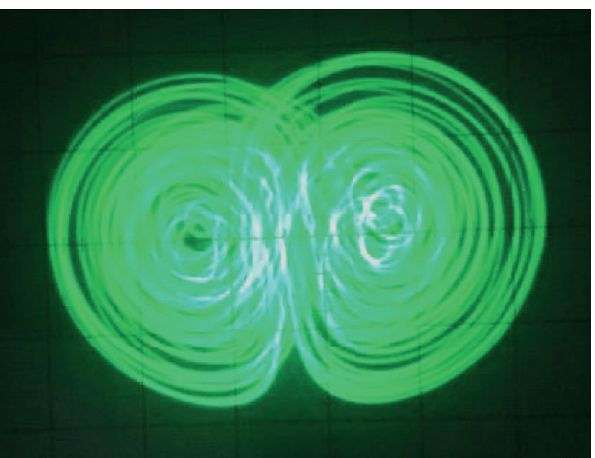

(b)

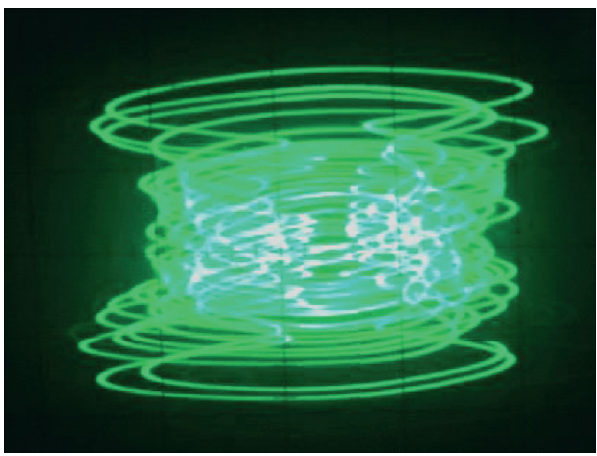

(d)

Figure 13: Continued. 


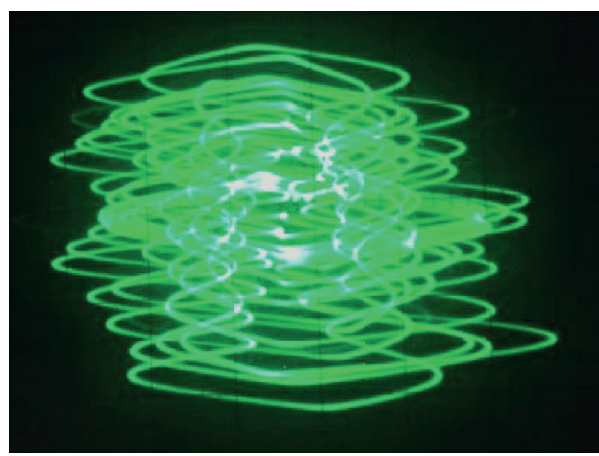

(e)

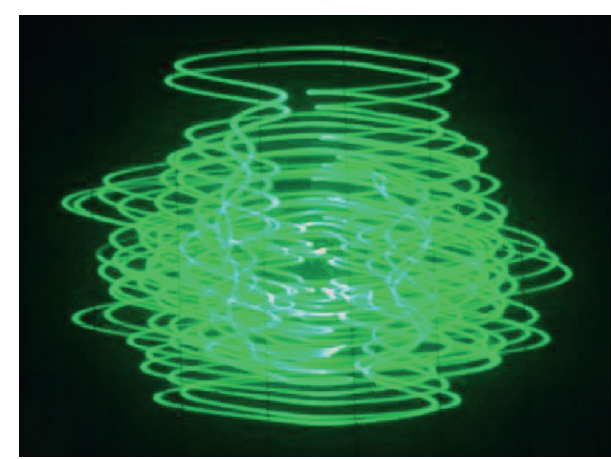

(f)

FIgURE 13: Experiment observations of the proposed 5D memristive hyperchaotic system: (a) $X$ - $Z$ plane, (b) $Y$ - $Z$ plane, (c) $X$ - $Y$ plane, (d) $X$ $W$ plane, (e) $Y$ - $W$ plane, (f) $Y-U$ plane.

$R_{8}=250 \mathrm{k} \Omega, R_{11}=5 \mathrm{M} \Omega, R_{12}=R_{14}=200 \mathrm{k} \Omega, R_{5}=R_{9}=10 \mathrm{k} \Omega$, and the value of the feedback resistance can be set as $R_{15}=R_{16}=R_{17}=R_{18}=R_{19}=R_{20}=R_{21}=10 \mathrm{k} \Omega, R_{22}=100 \mathrm{k} \Omega$.

The experimental results are captured by an analog oscilloscope (REK-620CH) as shown in Figure 13. Obviously, the experimental results are well consistent with the numerical simulations in Figure 1, which have verified the correctness and the physical realizability of this novel $5 \mathrm{D}$ memristive hyperchaotic system.

\section{Conclusion}

In this paper, by utilizing a memristor to substitute a linear coupling resistor and adding DC sources to realize two constant terms in the realization circuit of an improved 4D self-excited hyperchaotic system, a novel 5D memristive hyperchaotic system without equilibrium points is presented. The complex nonlinear dynamical behaviors of the novel 5D memristive hyperchaotic system are investigated under the variation of its system parameters and the memristor and other initial conditions, including hidden hyperchaotic attractors, hidden extreme multistability, and transient hyperchaotic behavior. More interestingly, by using a single constant, the freedom of offset boosting of a state variable of this system is obtained. Additionally, the hardware experiments are performed to verify numerical simulations. Since there is hidden extreme multistability in the proposed 5D memristive hyperchaotic system, it means that this system is especially suitable for engineering applications such as pseudorandom signal generation and encryption field.

\section{Data Availability}

The data used to support the findings of this study are available from the corresponding author upon request.

\section{Conflicts of Interest}

The authors declare that there are no conflicts of interest regarding the publication of this paper.

\section{Acknowledgments}

The authors would like to acknowledge the support provided by the National Natural Science Foundation of China (Grant nos. 61901169 and 61504013) and the Natural Science Foundation of Hunan Province, China (Grant nos. 2019JJ40190 and 2019JJ50648).

\section{References}

[1] E. N. Lorenz, "Deterministic nonperiodic flow," Journal of the Atmospheric Sciences, vol. 20, no. 2, pp. 130-141, 1963.

[2] I. Pehlivan, E. Kurt, Q. Lai, B. Aziz, and M. C. Kutlu, "A multiscroll chaotic attractor and its electronic circuit implementation," Chaos Theory and Applications, vol. 1, no. 1, pp. 29-37, 2019.

[3] Y. Adıyaman, S. Emiroglu, M. K. Uçar, and M. Yıldız, "Dynamical analysis, electronic circuit design and control application of a different chaotic system," Chaos Theory and Applications, vol. 2, no. 1, pp. 10-16, 2020.

[4] Q. Yang, D. Zhu, and L. Yang, "A new 7D hyperchaotic system with five positive Lyapunov exponents coined," International Journal of Bifurcation and Chaos, vol. 28, no. 05, p. $1850057,2018$.

[5] F. Nazarimehr, K. Rajagopal, J. Kengne, S. Jafari, and V.-T. Pham, "A new four-dimensional system containing chaotic or hyper-chaotic attractors with no equilibrium, a line of equilibria and unstable equilibria," Chaos, Solitons \& Fractals, vol. 111, pp. 108-118, 2018.

[6] Q. Yang and M. Bai, "A new 5D hyperchaotic system based on modified generalized Lorenz system," Nonlinear Dynamics, vol. 88, no. 1, pp. 189-221, 2017.

[7] Yu Fei, S. Qian, Xi Chen et al., "A new 4D four-wing memristive hyperchaotic system: dynamical analysis, electronic circuit design, shape synchronization and secure communication," International Journal of Bifurcation and Chaos, vol. 30, no. 10, p. 2050147, 2020.

[8] Yu Fei, Li Liu, H. Shen et al., "Multistability analysis, coexisting multiple attractors and FPGA implementation of YuWang four-wing chaotic system," Mathematical Problems in Engineering, vol. 2020, Article ID 7530976, 2020.

[9] O. S. Ojoniyi and A. N. Njah, "A 5D hyperchaotic sprott B system with coexisting hidden attractors," Chaos, Solitons \& Fractals, vol. 87, pp. 172-181, 2016. 
[10] S. Vaidyanathan, A. Akgul, S. Kaçar, and U. Çavuşoğlu, “A new 4-D chaotic hyperjerk system, its synchronization, circuit design and applications in RNG, image encryption and chaosbased steganography," The European Physical Journal Plus, vol. 133, p. 46, 2018.

[11] C. Chen, J. Chen, H. Bao, M. Chen, and B. Bao, "Coexisting multi-stable patterns in memristor synapse-coupled Hopfield neural network with two neurons," Nonlinear Dynamics, vol. 95, no. 4, pp. 3385-3399, 2019.

[12] Yu Fei, Li Liu, H. Shen et al., "Dynamic analysis, circuit design and synchronization of a novel 6D memristive four-wing hyperchaotic system with multiple coexisting attractors,"Complexity, vol. 2020, Article ID 5904607, 2020.

[13] F. Yu, Z. Wei, U. E. Kocamaz, A. Akif, and I. Moroz, "Synchronization and electronic circuit application of hidden hyperchaos in a four-dimensional self-exciting homopolar disc dynamo without equilibria," Complexity, vol. 2017, Article ID 7101927, 2017.

[14] R. L. Filali, M. Benrejeb, and P. Borne, "On observer-based secure communication design using discrete-time hyperchaotic systems," Communications in Nonlinear Science and Numerical Simulation, vol. 19, no. 5, pp. 1424-1432, 2014.

[15] X. Wang, A. Akgul, S. Kacar, and V.-T. Pham, "Multimedia security application of a ten-term chaotic system without equilibrium," Complexity, vol. 2017, Article ID 8412093, 2017.

[16] L. O. Chua, "Memristor-the missing circuit element," IEEE Transactions on Circuit Theory, vol. 18, no. 5, pp. 507-519, 1971.

[17] D. B. Strukov, G. S. Snider, D. R. Stewart, and R. S. Williams, "The missing memristor found," Nature, vol. 453, no. 7191, pp. 80-83, 2008.

[18] M. Chen, B. Bao, T. Jiang et al., "Flux-charge analysis of initial state-dependent dynamical behaviors of a memristor emulator-based chua's circuit," International Journal of Bifurcation and Chaos, vol. 28, no. 10, p. 1850120, 2018.

[19] B. C. Bao, P. Y. Wu, H. Bao, H. G. Wu, X. Zhang, and M. Chen, "Symmetric periodic bursting behavior and bifurcation mechanism in a third-order memristive diode bridgebased oscillator," Chaos, Solitons \& Fractals, vol. 109, pp. 146-153, 2018.

[20] S. Lee, J. Sohn, Z. Jiang et al., "Metal oxide-resistive memory using graphene-edge electrodes," Nature Communications, vol. 6, p. 8407, 2015

[21] A. Wedig, M. Luebben, D. Y. Cho et al., "Nanoscale cation motion in $\mathrm{TaO}(\mathrm{x}), \mathrm{HfO}(\mathrm{x})$ and $\mathrm{TiO}(\mathrm{x})$ memristive systems," Nature Nanotechnology, vol. 11, no. 1, pp. 67-74, 2016.

[22] Q. Xu, Z. Song, H. Bao, M. Chen, and B. Bao, "Two-neuronbased non-autonomous memristive Hopfield neural network: numerical analyses and hardware experiments," AEU - International Journal of Electronics and Communications, vol. 96, no. 11, pp. 66-74, 2018.

[23] S. Wang, C. Wang, and C. Xu, "An image encryption algorithm based on a hidden attractor chaos system and the Knuth-Durstenfeld algorithm," Optics and Lasers in Engineering, vol. 128, p. 105995, 2020.

[24] H. Lin, C. Wang, Y. Sun, and W. Yao, "Firing multistability in a locally active memristive neuron model," Nonlinear Dynamics, vol. 100, no. 4, pp. 3667-3683, 2020.

[25] H. Lin, C. Wang, and Y. Tan, "Hidden extreme multistability with hyperchaos and transient chaos in a Hopfield neural network affected by electromagnetic radiation," Nonlinear Dynamics, vol. 99, no. 3, pp. 2369-2386, 2020.

[26] B. A. Mezatio, M. M. Tingue, R. Kengne et al., "Complex dynamics from a novel memristive $6 \mathrm{D}$ hyperchaotic autonomous system," International Journal of Dynamics and Control, vol. 8, pp. 70-90, 2020.

[27] J. Ma, Z. Chen, Z. Wang, and Q. Zhang, "A four-wing hyperchaotic attractor generated from a 4-D memristive system with a line equilibrium," Nonlinear Dynamics, vol. 81, no. 3, pp. 1275-1288, 2015.

[28] A. Sambas, S. Vaidyanathan, S. Zhang, Y. Zeng, M. A. Mohamed, and M. Mamat, "A new double-wing chaotic system with coexisting attractors and line equilibrium: bifurcation analysis and electronic circuit simulation," IEEE Access, vol. 7, pp. 115454-115462, 2019.

[29] A. Sambas, S. Vaidyanathan, E. Tlelo-Cuautle et al., "A novel chaotic system with two circles of equilibrium points: multistability, electronic circuit and FPGA realization," Electronics, vol. 8, no. 11, p. 1211, 2019.

[30] A. Sambas, S. Vaidyanathan, S. Zhang et al., "Multistability in a novel chaotic system with perpendicular lines of equilibrium: analysis, adaptive synchronization and circuit design," Engineering Letters, vol. 27, no. 4, pp. 744-751, 2019.

[31] C. Wang, H. Xia, and L. Zhou, "Implementation of a new memristor-based multiscroll hyperchaotic system," Pramana, vol. 88, no. 2, p. 34, 2017.

[32] S. Zhang and Y. Zeng, "A simple Jerk-like system without equilibrium: asymmetric coexisting hidden attractors, bursting oscillation and double full feigenbaum remerging trees," Chaos, Solitons \& Fractals, vol. 120, pp. 25-40, 2019.

[33] S. Jafari, J. C. Sprott, and F. Nazarimehr, "Recent new examples of hidden attractors," The European Physical Journal Special Topics, vol. 224, no. 8, pp. 1469-1476, 2015.

[34] F. Nazarimehr, B. Saedi, S. Jafari, and J. C. Sprott, "Are perpetual points sufficient for locating hidden attractors?" International Journal of Bifurcation and Chaos, vol. 27, no. 03, p. 1750037, 2017.

[35] M. N. Zavareh, F. Nazarimehr, K. Rajagopal, and S. Jafari, "Hidden attractor in a passive motion model of compass-gait robot," International Journal of Bifurcation and Chaos, vol. 28, no. 14, p. 1850171, 2018.

[36] X. Wang and G. Chen, "A chaotic system with only one stable equilibrium," Communications in Nonlinear Science and Numerical Simulation, vol. 17, no. 3, pp. 1264-1272, 2012.

[37] V.-T. Pham, S. Jafari, T. Kapitaniak et al., "Generating a chaotic system with one stable equilibrium," International Journal of Bifurcation and Chaos, vol. 27, no. 4, Article ID 1750053, 2017.

[38] V.-T. Pham, S. Jafari, C. Volos et al., "A gallery of chaotic systems with an infinite number of equilibrium points," Chaos, Solitons and Fractals, vol. 93, pp. 58-63, 2016.

[39] S. Jafari, J. C. Sprott, V.-T. Pham, C. Volos, and C. Li, "Simple chaotic 3D flows with surfaces of equilibria," Nonlinear Dynamics, vol. 86, no. 2, pp. 1349-1358, 2016.

[40] W. Zhou, G. Wang, Y. Shen, F. Yuan, and S. Yu, "Hidden coexisting attractors in a chaotic system without equilibrium point," International Journal of Bifurcation and Chaos, vol. 28, no. 10, p. 1830033, 2018.

[41] S. Zhang, Y. Zeng, and Z. Li, "A novel four-dimensional Noequilibrium hyper-chaotic system with grid multiwing hyperchaotic hidden attractors," Journal of Computational and Nonlinear Dynamics, vol. 13, no. 9, Article ID 090908, 2018.

[42] D. Dudkowski, A. Prasad, and T. Kapitaniak, "Perpetual points and hidden attractors in dynamical systems," Physics Letters A, vol. 379, no. 40-41, pp. 2591-2596, 2015.

[43] A. Akgul, H. Calgan, I. Koyuncu, I. Pehlivan, and A. Istanbullu, "Chaos-based engineering applications with a 
3D chaotic system without equilibrium points," Nonlinear Dynamics, vol. 84, no. 2, pp. 481-495, 2016.

[44] S. Jafari and J. C. Sprott, "Simple chaotic flows with a line equilibrium," Chaos, Solitons \& Fractals, vol. 57, pp. 79-84, 2013.

[45] G. Wang, F. Yuan, G. Chen, and Yu Zhang, "Coexisting multiple attractors and riddled basins of a memristive system," Chaos, vol. 28, Article ID 013125, 2018.

[46] X. Zhang and Z. Li, "Hidden extreme multistability in a novel 4D fractional-order chaotic system," International Journal of Non-linear Mechanics, vol. 111, pp. 14-27, 2019.

[47] C. Li, J. C. Sprott, W. Hu, and Y. Xu, "Infinite multistability in a self-reproducing chaotic system," International Journal of Bifurcation and Chaos, vol. 27, no. 10, p. 1750160, 2017.

[48] B. Han, N. Wang, B. Bao, M. Chen, P. Jin, and G. Wang, "Initial condition-dependent dynamics and transient period in memristor-based hypogenetic jerk system with four line equilibria," Communications in Nonlinear Science and $\mathrm{Nu}$ merical Simulation, vol. 57, pp. 264-275, 2018.

[49] B. A. Mezatio, M. T. Motchongom, and B. W. Tekam, "A novel memristive 6D hyperchaotic autonomous system with hidden extreme multistability," Chaos, Solitons and Fractals, vol. 120, pp. 100-115, 2019.

[50] F. Yuan, G. Wang, and X. Wang, "Extreme multistability in a memristor-based multi-scroll hyper-chaotic system," Chaos, vol. 26, no. 7, Article ID 073107, 2016.

[51] J. Kengne, Z. Njitacke Tabekoueng, V. Kamdoum Tamba, and A. Nguomkam Negou, "Periodicity, chaos, and multiple attractors in a memristor-based Shinriki's circuit," Chaos: An Interdisciplinary Journal of Nonlinear Science, vol. 25, no. 10, p. 103126, 2015.

[52] S. Morfu, B. Nofiele, and P. Marquié, "On the use of multistability for image processing," Physics Letters A, vol. 367, no. 3, pp. 192-198, 2007.

[53] L. Zhou, Z. Chen, J. Wang, and Q. Zhang, "Local bifurcation analysis and global dynamics estimation of a novel 4-dimensional hyperchaotic system," International Journal of Bifurcation and Chaos, vol. 27, no. 02, p. 1750021, 2017.

[54] J. Kengne, G. D. Leutcho, and A. N. K. Telem, "Reversals of period doubling, coexisting multiple attractors, and offset boosting in a novel memristive diode bridge-based hyperjerk circuit," Analog Integrated Circuits and Signal Processing, vol. 101, no. 3, pp. 379-399, 2019.

[55] H. G. Wu, Y. Ye, B. C. Bao, M. Chen, and Q. Xu, "Memristor initial boosting behaviors in a two-memristor-based hyperchaotic system," Chaos, Solitons \& Fractals, vol. 121, pp. 178-185, 2019. 